\title{
Lepto-quark portal dark matter
}

\author{
Soo-Min Choi, ${ }^{a}$ Yoo-Jin Kang, ${ }^{a}$ Hyun Min Lee ${ }^{a, b}$ and Tae-Gyu Ro ${ }^{a}$ \\ ${ }^{a}$ Department of Physics, Chung-Ang University, \\ Seoul 06974, Korea \\ ${ }^{b}$ School of Physics, Korea Institute for Advanced Study, \\ Seoul 02455, Korea \\ E-mail: soominchoi90@gmail.com, yoojinkang91@gmail.com, \\ hminlee@cau.ac.kr, shxorb234@gmail.com
}

ABSTRACT: We consider the extension of the Standard Model with scalar leptoquarks as a portal to dark matter (DM), motivated by the recent anomalies in semi-leptonic $B$-meson decays. Taking singlet and triplet scalar leptoquarks as the best scenarios for explaining $B$-meson anomalies, we discuss the phenomenological constraints from rare meson decays, muon $(g-2)_{\mu}$, and leptoquark searches at the Large Hadron Collider (LHC). Introducing leptoquark couplings to scalar dark matter, we find that the DM annihilations into a pair of leptoquarks open a wide parameter space, being compatible with XENON1T bound, and show that there is an interesting interplay between LHC leptoquark searches and distinct signatures from cascade annihilations of dark matter.

Keywords: Beyond Standard Model, Cosmology of Theories beyond the SM, Heavy Quark Physics

ARXIV EPRINT: 1807.06547 


\section{Contents}

1 Introduction 1

2 Overview on $R_{K^{(*)}}$ and $R_{D^{(*)}}$ anomalies 3

3 Leptoquarks for $B$-meson anomalies $\quad 4$

3.1 Effective interactions from scalar leptoquarks 4

3.2 Singlet scalar leptoquark 5

$\begin{array}{lll}3.3 & \text { Triplet scalar leptoquark } & 6\end{array}$

4 Constraints on leptoquarks $\quad 7$

4.1 Rare meson decays and mixing $\quad 7$

$\begin{array}{llr}4.2(g-2)_{\mu} & 9\end{array}$

$\begin{array}{lll}4.3 & \text { Leptoquark searches } & 10\end{array}$

5 Leptoquarks and scalar dark matter $\quad 10$

5.1 Annihilation cross sections for scalar dark matter 11

$\begin{array}{lll}5.2 & \text { Direct detection bounds } & 14\end{array}$

$\begin{array}{lll}5.3 & \text { Indirect detection bounds } & 15\end{array}$

$\begin{array}{ll}5.4 \text { Higgs data } & 20\end{array}$

6 Conclusions 20

A Effective Hamiltonians for $B$-meson decays $\quad 21$

B Effective interactions for dark matter and Higgs boson due to leptoquark loops 23

\section{Introduction}

Recently, there have been intriguing anomalies in the semi-leptonic decays of $B$-mesons at BaBar, Belle and LHCb experiments, which are based on the observables of testing Lepton Flavor Universality(LFU), i.e. $R_{K^{(*)}}[1-6]$ and $R_{D^{(*)}}$ [7-11]. Thus, it is plausible that LFU might be violated due to new physics in the neutral and charged currents associated with muon and tau leptons, respectively. Currently, experimental values of $R_{K^{(*)}}$ and $R_{D^{(*)}}$ turn out to be deviated from the SM expectations at about $4 \sigma$ level per each. However, we still need to understand the hadronic uncertainties in angular distributions of related $B$-meson decays [12-15] and the results are to be confirmed at LHCb with more data and Belle II [16]. Nonetheless, it is important to study the consequences of new physics in direct searches at the LHC and other precision and indirect observables. 
Dark matter (DM) is known to occupy about $85 \%$ of the total matter density in the Universe, and there are a variety of evidences for the existence of dark matter such as galaxy rotation curves, gravitational lensing, large scale structure, etc. The Weakly Interacting Massive Particles (WIMPs) paradigm has driven forces for searching particle dark matter with non-gravitational interactions beyond the Standard Model (SM) for more than three decades. Various direct detection experiments [17-21] have put stringent bounds on the cross section of DM-nucleon elastic scattering, and forthcoming XENON-nT and largescale experiments such as DARWIN [22] and LZ [23] will push the limits further to the neutrino floor where there are irreducible backgrounds due to neutrino coherent scattering. In particular, Higgs-portal type models for dark matter have been strongly constrained, apart from the resonance region or the heavy DM masses.

Leptoquark models $[24,25]$ have been revived recently because they can provide an economic way of accommodating the aforementioned $B$-meson anomalies [26-32] and can be tested at the LHC. Leptoquarks carry extra Yukawa-type couplings to the SM fermions, providing a source for violating LFU. Furthermore, leptoquark scalars or vectors could be originated from unified models of forces [33-38], in analogy to colored triplet Higgs scalars or $X, Y$ gauge bosons in the minimal $\mathrm{SU}(5)$ unification. The best scenarios for explaining the $B$-meson anomalies $[27,28]$ are: one $\mathrm{SU}(2)_{L^{-}}$-singlet scalar leptoquark $S_{1}$ for $R_{D^{(*)}}$, and one $\mathrm{SU}(2)_{L^{-}}$triplet scalar leptoquark $S_{3}$ for $R_{K^{(*)}}$, or one $\mathrm{SU}(2)_{L^{-}}$-singlet vector leptoquark for both $B$-meson anomalies. Leptoquark scenarios are phenomenologically rich, because the muon $(g-2)_{\mu}$ anomalies can be also explained by leptoquark couplings and various LHC searches can be reinterpreted to bound the leptoquark models.

In this article, we consider a leptoquark-portal model for dark matter where scalar dark matter communicates with the SM through the quartic couplings of scalar leptoquarks, $S_{1}$ and $S_{3}$. We show that sizable leptoquark couplings to dark matter lead to new annihilation channels of dark matter into a pair of leptoquarks, opening a wide parameter space where the correct relic density can be explained, being compatible with the direct detection bounds from XENON1T. Moreover, we also discuss that the cascade annihilations of dark matter can lead to distinct signatures for cosmic ray observation, in correlation to leptoquark searches at the LHC. We argue that our models with scalar leptoquarks are consistent with the current bounds from rare meson decays, mixings and lepton flavor violation, whereas the loop corrections of leptoquarks to DM-nucleon couplings and Higgs couplings can be negligible in most of the parameter space of our interest.

The paper is organized as follows. We first give a brief overview on the $R_{K^{(*)}}$ and $R_{D^{(*)}}$ anomalies and the necessary corrections to the effective Hamiltonians. Then, in models with scalar leptoquarks, we derive the effective interactions for the semi-leptonic $B$-meson decays and discuss the conditions for $B$-meson anomalies and various constraints from rare meson decays, mixings, muon $(g-2)_{\mu}$ and leptoquark searches at the LHC. Next we describe leptoquark-portal models for dark matter and consider various constraints on the models, coming from the relic density, direct and indirect detection of dark matter and Higgs data. There are two appendices dealing with the details on effective Hamiltonians for $B$-meson decays and effective interactions for dark matter and Higgs due to leptoquarks, respectively. Finally, conclusions are drawn. 


\section{Overview on $\boldsymbol{R}_{K^{(*)}}$ and $\boldsymbol{R}_{D^{(*)}}$ anomalies}

In this section, we give a brief overview on the status of the $B$-meson anomalies and the interpretations in terms of the effective Hamiltonians in the SM.

The reported value of $R_{K}=\mathcal{B}\left(B \rightarrow K \mu^{+} \mu^{-}\right) / \mathcal{B}\left(B \rightarrow K e^{+} e^{-}\right)[1]$ is

$$
R_{K}=0.745_{-0.074}^{+0.090} \text { (stat) } \pm 0.036 \text { (syst) }, \quad 1 \mathrm{GeV}^{2}<q^{2}<6 \mathrm{GeV}^{2},
$$

which deviates from the SM prediction by $2.6 \sigma$. On the other hand for vector $B$-mesons, $R_{K^{*}}=\mathcal{B}\left(B \rightarrow K^{*} \mu^{+} \mu^{-}\right) / \mathcal{B}\left(B \rightarrow K^{*} e^{+} e^{-}\right)[2-4]$ is

$$
\begin{array}{lr}
R_{K^{*}}=0.66_{-0.07}^{+0.11} \text { (stat) } \pm 0.03 \text { (syst) }, & 0.045 \mathrm{GeV}^{2}<q^{2}<1.1 \mathrm{GeV}^{2}, \\
R_{K^{*}}=0.69_{-0.07}^{+0.11} \text { (stat) } \pm 0.05 \text { (syst), } & 1.1 \mathrm{GeV}^{2}<q^{2}<6.0 \mathrm{GeV}^{2},
\end{array}
$$

which again differs from the SM prediction by $2.1-2.3 \sigma$ and $2.4-2.5 \sigma$, depending on the energy bins. The deviation in $R_{K^{*}}$ is supported by the reduction in the angular distribution of $B \rightarrow K^{*} \mu^{+} \mu^{-}$, the so called $P_{5}^{\prime}$ variable $[5,6]$.

The effective Hamiltonian for $b \rightarrow s \mu^{+} \mu^{-}$is given by

$$
\Delta \mathcal{H}_{\mathrm{eff}, \bar{b} \rightarrow \bar{s} \mu^{+} \mu^{-}}=-\frac{4 G_{F}}{\sqrt{2}} V_{t s}^{*} V_{t b} \frac{\alpha_{e m}}{4 \pi}\left(C_{9}^{\mu} \mathcal{O}_{9}^{\mu}+C_{10}^{\mu} \mathcal{O}_{10}^{\mu}+C_{9}^{\prime \mu} \mathcal{O}_{9}^{\prime \mu}+C_{10}^{\prime \mu} \mathcal{O}_{10}^{\prime \mu}\right)+\text { h.c. }
$$

where $\mathcal{O}_{9}^{\mu} \equiv\left(\bar{s} \gamma^{\mu} P_{L} b\right)\left(\bar{\mu} \gamma_{\mu} \mu\right), \mathcal{O}_{10}^{\mu} \equiv\left(\bar{s} \gamma^{\mu} P_{L} b\right)\left(\bar{\mu} \gamma_{\mu} \gamma^{5} \mu\right), \mathcal{O}_{9}^{\prime \mu} \equiv\left(\bar{s} \gamma^{\mu} P_{R} b\right)\left(\bar{\mu} \gamma_{\mu} \mu\right)$ and $\mathcal{O}_{10}^{\prime \mu} \equiv$ $\left(\bar{s} \gamma^{\mu} P_{R} b\right)\left(\bar{\mu} \gamma_{\mu} \gamma^{5} \mu\right)$, and $\alpha_{\text {em }}$ is the electromagnetic coupling. In the SM, the Wilson coefficients are given by $C_{9}^{\mu, \mathrm{SM}}\left(m_{b}\right)=-C_{10}^{\mu, \mathrm{SM}}\left(m_{b}\right)=4.27$ and $C_{9}^{\prime \mu, \mathrm{SM}}\left(m_{b}\right) \approx-C_{10}^{\prime \mu, \mathrm{SM}}\left(m_{b}\right) \approx 0$.

For $C_{10}^{\mu, \mathrm{NP}}=C_{9}^{\prime \mu, \mathrm{NP}}=C_{10}^{\prime \mu, \mathrm{NP}}=0$, the best-fit value for new physics contribution is given by $C_{9}^{\mu, \mathrm{NP}}=-1.11$ [39], (while taking $[-1.28,-0.94]$ and $[-1.45,-0.75]$ within $1 \sigma$ and $2 \sigma$ errors), to explain the $R_{K^{(*)}}$ anomalies. On the other hand, for $C_{9}^{\mu, \mathrm{NP}}=-C_{10}^{\mu, \mathrm{NP}}$ and others being zero, the best-fit value for new physics contribution is given by $C_{9}^{\mu, \mathrm{NP}}=$ -0.62 [39], (while taking $[-0.75,-0.49]$ and $[-0.88,-0.37]$ within $1 \sigma$ and $2 \sigma$ errors).

Taking the results of BaBar [7, 8], Belle $[9,10]$ and LHCb [11] for $R_{D}=\mathcal{B}(B \rightarrow$ $D \tau \nu) / \mathcal{B}(B \rightarrow D l \nu)$ and $R_{D^{*}}=\mathcal{B}\left(B \rightarrow D^{*} \tau \nu\right) / \mathcal{B}\left(B \rightarrow D^{*} l \nu\right)$ with $l=e, \mu$ for BaBar and Belle and $l=\mu$ for LHCb, the Heavy Flavor Averaging Group [40] reported the experimental world averages as follows,

$$
\begin{aligned}
& R_{D}^{\exp }=0.403 \pm 0.040 \pm 0.024 \\
& R_{D^{*}}^{\exp }=0.310 \pm 0.015 \pm 0.008 .
\end{aligned}
$$

On the other hand, taking into account the lattice calculation of $R_{D}$, which is $R_{D}=$ $0.299 \pm 0.011$ [41], and the uncertainties in $R_{D^{*}}$ in various groups [42-47], we take the SM predictions for these ratios as follows,

$$
\begin{aligned}
& R_{D}^{\mathrm{SM}}=0.299 \pm 0.011 \\
& R_{D^{*}}^{\mathrm{SM}}=0.260 \pm 0.010
\end{aligned}
$$


Then, the combined derivation between the measurements and the SM predictions for $R_{D}$ and $R_{D^{*}}$ is about $4.1 \sigma$. We quote the best fit values for $R_{D}$ and $R_{D^{*}}$ including the new physics contributions [48],

$$
\frac{R_{D}}{R_{D}^{\mathrm{SM}}}=\frac{R_{D^{*}}}{R_{D^{*}}^{\mathrm{SM}}}=1.21 \pm 0.06
$$

The effective Hamiltonian for $b \rightarrow c \tau \nu$ in the SM is given by

$$
\mathcal{H}_{\mathrm{eff}}=\frac{4 G_{F}}{\sqrt{2}} V_{c b} C_{c b}\left(\bar{c} \gamma^{\mu} P_{L} b\right)\left(\bar{\tau} \gamma_{\mu} P_{L} \nu_{\tau}\right)+\text { h.c. }
$$

where $C_{c b}=1$ in the SM with $V_{c b} \approx 0.04$. The new physics contribution may contain the dimension-6 four-fermion vector operators, $\mathcal{O}_{V_{R, L}}=\left(\bar{c} \gamma^{\mu} P_{R, L} b\right)\left(\bar{\tau} \gamma_{\mu} P_{L} \nu_{\tau}\right)$ and/or scalar operators, $\mathcal{O}_{S_{R, L}}=\left(\bar{c} P_{R, L} b\right)\left(\bar{\tau} P_{L} \nu_{\tau}\right)$. Then, in order to explain the $R_{D^{(*)}}$ anomalies in eq. (2.8), the Wilson coefficient for the new physics contribution should be $\Delta C_{c b}=0.1$ from eq. (2.9), while taking [0.072, 0.127] and [0.044, 0.153] within $1 \sigma$ and $2 \sigma$ errors.

\section{Leptoquarks for $B$-meson anomalies}

It is known that $\mathrm{SU}(2)_{L}$ singlet and triplet scalar leptoquarks can explain $R_{D^{(*)}}$ and $R_{K^{(*)}}$ anomalies, respectively [27, 28]. (See also refs. [26, 29, 30, 32].) Thus, in this section, focusing on those scalar leptoquark models, we discuss the phenomenological constraints coming from the $B$-meson anomalies.

\subsection{Effective interactions from scalar leptoquarks}

We consider the Lagrangian for an $\mathrm{SU}(2)_{L}$ singlet scalar leptoquark $S_{1}$ with $Y=+\frac{1}{3}$, and an $\mathrm{SU}(2)_{L}$ triplet scalar leptoquark, $S_{3} \equiv \Phi_{a b}$ with $Y=+\frac{1}{3}$, as follows,

$$
\begin{aligned}
\mathcal{L}_{L Q} & =\mathcal{L}_{S_{1}}+\mathcal{L}_{S_{3}} \\
\mathcal{L}_{S_{1}} & =-\lambda_{i j} Q_{L i}^{a}\left(i \sigma^{2}\right)_{a b} S_{1} L_{L j}^{b}+\text { h.c. } \\
& =-\lambda_{i j} \overline{\left(Q^{C}\right)_{R i}^{a}}\left(i \sigma^{2}\right)_{a b} S_{1} L_{L j}^{b}+\text { h.c. }
\end{aligned}
$$

where $a, b$ are $\mathrm{SU}(2)_{L}$ indices, $\sigma^{2}$ is the second Pauli matrix and $\psi^{C}=C \bar{\psi}^{T}$ is the charge conjugate with $C=i \gamma^{0} \gamma^{2}$, and

$$
\begin{aligned}
\mathcal{L}_{S_{3}} & =-\kappa_{i j} Q_{L i}^{a} \Phi_{a b} L_{L j}^{b}+\text { h.c. } \\
& =-\kappa_{i j} \overline{\left(Q^{C}\right)_{R i}^{a}} \Phi_{a b} L_{L j}^{b}+\text { h.c. }
\end{aligned}
$$

with

$$
\Phi_{a b}=\left(\begin{array}{cc}
\sqrt{2} \phi_{3} & -\phi_{2} \\
-\phi_{2} & -\sqrt{2} \phi_{1}
\end{array}\right)
$$

where $\left(\phi_{1}, \phi_{2}, \phi_{3}\right)$ forms an isospin triplet with $T_{3}=+1,0,-1$ and $Q=+\frac{4}{3},+\frac{1}{3},-\frac{2}{3}$. We note that our conventions are comparable to those in the literature by writing $\Phi=$ $\left(i \sigma^{2}\right)(\vec{\sigma} \cdot \vec{S})$ where $\vec{\sigma}$ are Pauli matrices and $\vec{S}$ are complex scalar fields. 
Then, after integrating out the leptoquark scalars, we obtain the effective Lagrangian for the SM fermions in the following,

$$
\begin{aligned}
\mathcal{L}_{\mathrm{eff}}= & \left(\frac{1}{4 m_{S_{1}}^{2}} \lambda_{i j} \lambda_{k l}^{*}+\frac{3}{4 m_{S_{3}}^{2}} \kappa_{i j} \kappa_{k l}^{*}\right)\left(\bar{Q}_{L k} \gamma^{\mu} Q_{L i}\right)\left(\bar{L}_{L l} \gamma_{\mu} L_{L j}\right) \\
& +\left(-\frac{1}{4 m_{S_{1}}^{2}} \lambda_{i j} \lambda_{k l}^{*}+\frac{1}{4 m_{S_{3}}^{2}} \kappa_{i j} \kappa_{k l}^{*}\right)\left(\bar{Q}_{L k} \gamma^{\mu} \sigma^{I} Q_{L i}\right)\left(\bar{L}_{L l} \gamma_{\mu} \sigma^{I} L_{L j}\right)
\end{aligned}
$$

where $\sigma^{I}(I=1,2,3)$ are the Pauli matrices. There, we find that there are both $\mathrm{SU}(2)_{L}$ singlet and triplet $V-A$ operators. As compared to the case with $\mathrm{U}(2)$ flavor symmetry [28], the effective interactions for either singlet or triplet leptoquark can be written as

$$
\mathcal{L}_{\text {eff }}=-\frac{1}{v^{2}} \lambda_{k i}^{q} \lambda_{l j}^{l}\left[C_{S}\left(\bar{Q}_{L k} \gamma^{\mu} Q_{L i}\right)\left(\bar{L}_{L l} \gamma_{\mu} L_{L j}\right)+C_{T}\left(\bar{Q}_{L k} \gamma^{\mu} \sigma^{I} Q_{L i}\right)\left(\bar{L}_{L l} \gamma_{\mu} \sigma^{I} L_{L j}\right)\right]
$$

So, we obtain $C_{S}=-C_{T}$ for the singlet leptoquark and $C_{S}=3 C_{T}$ for the triplet leptoquark. A fit to low-energy data including the $R_{K^{(*)}}$ and $R_{D^{(*)}}$ anomalies has been done with four free parameters, $C_{T}, C_{S}, \lambda_{s b}^{q}$ and $\lambda_{\mu \mu}^{l}$, under the assumption that the CKM matrix stems solely from the mixing between up-type quarks [28]. As a result, the best-fit values are given by $C_{S} \approx C_{T} \approx 0.02$ for $\left|\lambda_{s b}^{q}\right|<5\left|V_{c b}\right|[28]$.

\subsection{Singlet scalar leptoquark}

After integrating out the leptoquark $S_{1}$, from the results in eq. (A.2), we obtain the effective Hamiltonian relevant for $b \rightarrow c \tau \bar{\nu}_{\tau}$ as

$$
\mathcal{H}_{b \rightarrow c \tau \bar{\nu}_{\tau}}^{S_{1}}=-\frac{\lambda_{33}^{*} \lambda_{23}}{2 m_{S_{1}}^{2}}\left(\bar{b}_{L} \gamma^{\mu} c_{L}\right)\left(\bar{\nu}_{\tau L} \gamma_{\mu} \tau_{L}\right)+\text { h.c. } \equiv \frac{1}{\Lambda_{D}^{2}}\left(\bar{b}_{L} \gamma^{\mu} c_{L}\right)\left(\bar{\nu}_{\tau L} \gamma_{\mu} \tau_{L}\right)+\text { h.c. }
$$

As a consequence, the singlet leptoquark gives rise to the effective operator for explaining the $R_{D^{(*)}}$ anomalies and the effective cutoff scale is to be $\Lambda_{D} \sim 3.5 \mathrm{TeV}$ [49]. Thus, for $m_{S_{1}} \gtrsim 1 \mathrm{TeV}$, we need $\sqrt{\lambda_{33}^{*} \lambda_{23}} \gtrsim 0.4$.

In the left plot of figure 1 , we depict the parameter space for $m_{S_{1}}$ and the effective leptoquark coupling, $\lambda_{\text {eff }}=\sqrt{\left|\lambda_{33}^{*} \lambda_{23}\right|}$, in which the $R_{D^{(*)}}$ anomalies can be explained within $2 \sigma(1 \sigma)$ errors in green(yellow) region from the conditions below eq. (2.9).

From the couplings of the singlet scalar leptoquark necessary for $R_{D^{(*)}}$ anomalies,

$$
\begin{aligned}
\mathcal{L}_{S_{1}} \supset & -\lambda_{33}\left(\overline{\left(t^{C}\right)_{R}} S_{1} \tau_{L}-\overline{\left(b^{C}\right)_{R}} S_{1} \nu_{\tau L}\right)+\text { h.c. } \\
& -\lambda_{23}\left(\overline{\left(c^{C}\right)_{R}} S_{1} \tau_{L}-\overline{\left(s^{C}\right)_{R}} S_{1} \nu_{\tau L}\right)+\text { h.c. }
\end{aligned}
$$

the decay modes of the singlet scalar leptoquark are given by $S_{1} \rightarrow \bar{t} \bar{\tau}, \bar{c} \bar{\tau}$ and $S_{1} \rightarrow \bar{b} \nu_{\tau}, \bar{s} \nu_{\tau}$, which are summarized together with the corresponding LHC bounds on leptoquark masses in table 1. 

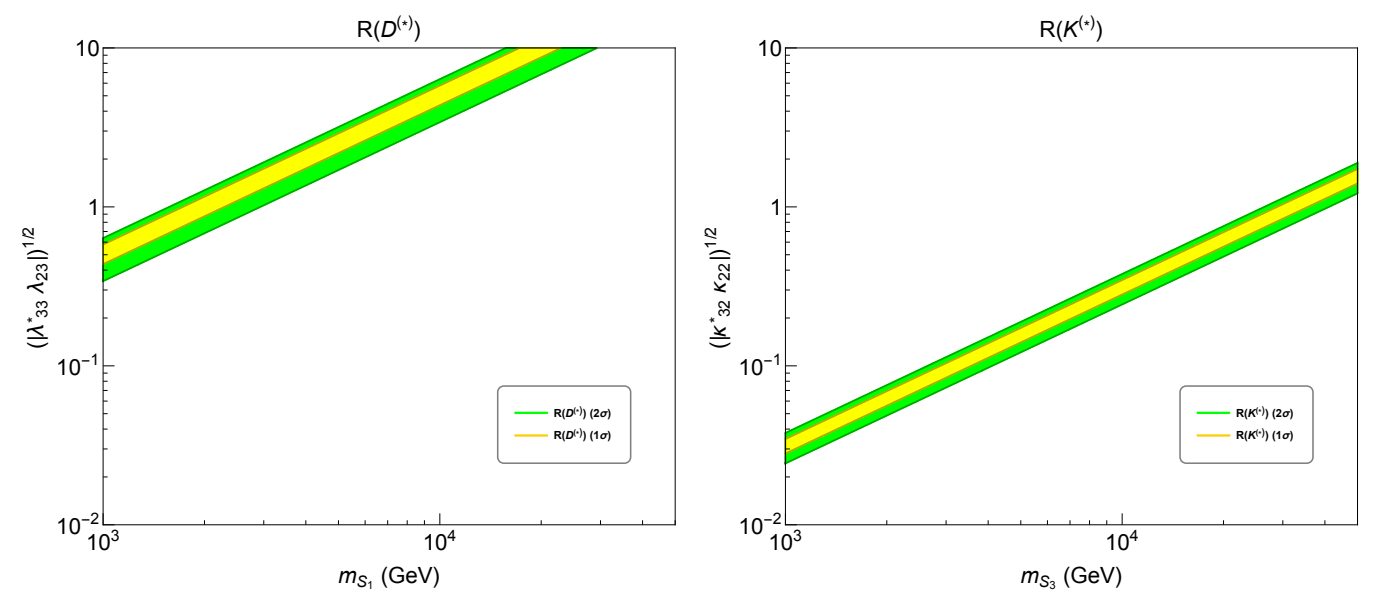

Figure 1. Parameter space for the leptoquark mass $m_{L Q}$ and the effective coupling $\lambda_{\text {eff }}$, explaining the B-meson anomalies, in green(yellow) region at $2 \sigma(1 \sigma)$ level. We have taken $m_{L Q}=m_{S_{1}}$ and $\lambda_{\text {eff }}=\sqrt{\left|\lambda_{33}^{*} \lambda_{23}\right|}$ for $R_{D^{(*)}}$ on left plot, and $m_{L Q}=m_{S_{3}}$ and $\lambda_{\text {eff }}=\sqrt{\left|\kappa_{32}^{*} \kappa_{22}\right|}$ for $R_{K^{(*)}}$ on right plot.

\begin{tabular}{|c|c|c|c|c|}
\hline LQs & BRs & $m_{L Q, \text { min }}$ & BRs & $m_{L Q, \min }$ \\
\hline$S_{1}$ & $\mathrm{~B}\left(\bar{t} \bar{\tau} / b \nu_{\tau}\right)=\frac{1}{2} \beta$ & $1.22 \mathrm{TeV}\left(b \nu_{\tau}\right)[50,51]$ & $\mathrm{B}\left(\bar{c} \bar{\tau} / s \nu_{\tau}\right)=\frac{1}{2}(1-\beta)$ & $950 \mathrm{GeV}\left(\nu_{\tau} j\right)[52,53]$ \\
\hline$S_{3}\left(\phi_{1}\right)$ & $\mathrm{B}(\bar{b} \bar{\mu})=\gamma$ & $1.4 \mathrm{TeV}[54]$ & $\mathrm{B}(\bar{s} \bar{\mu})=1-\gamma$ & $1.08 \mathrm{TeV}(\bar{\mu} j)[55]$ \\
\hline$S_{3}\left(\phi_{2}\right)$ & $\mathrm{B}\left(\bar{t} \bar{\mu} / \bar{b} \bar{\nu}_{\mu}\right)=\frac{1}{2} \gamma$ & $1.45 \mathrm{TeV}(\bar{t} \bar{\mu})[56]$ & $\mathrm{B}\left(\bar{c} \bar{\mu} / \bar{s} \bar{\nu}_{\mu}\right)=\frac{1}{2}(1-\gamma)$ & $850 \mathrm{GeV}\left(\bar{\mu} \bar{\nu}_{\mu} j j\right)[57]$ \\
\hline$S_{3}\left(\phi_{3}\right)$ & $\mathrm{B}\left(\bar{t} \bar{\nu}_{\mu}\right)=\gamma$ & $1.12 \mathrm{TeV}[58,59]$ & $\mathrm{B}\left(\bar{c} \bar{\nu}_{\mu}\right)=1-\gamma$ & $950 \mathrm{GeV}\left(\bar{\nu}_{\mu} j\right)[52,53]$ \\
\hline
\end{tabular}

Table 1. Decay branching ratios of leptoquarks, and LHC bounds on leptoquark masses. Here, $\beta \equiv \lambda_{33}^{2} /\left(\lambda_{33}^{2}+\lambda_{23}^{2}\right)$ and $\gamma \equiv \kappa_{32}^{2} /\left(\kappa_{32}^{2}+\kappa_{22}^{2}\right)$. Most LHC bounds are given for $B=1$, except in ref. [57] where $B(\bar{c} \bar{\mu})=B\left(\bar{s} \bar{\nu}_{\mu}\right)=0.5$ was taken.

\subsection{Triplet scalar leptoquark}

After integrating out the leptoquark $\phi_{1}$ with $Q=+\frac{4}{3}$, from the results in eq. (A.8), we also obtain the effective Hamiltonian relevant for $b \rightarrow s \mu^{+} \mu^{-}$as

$$
\mathcal{H}_{b \rightarrow s \mu^{+} \mu^{-}}^{S_{3}}=-\frac{\kappa_{32}^{*} \kappa_{22}}{m_{\phi_{1}}^{2}}\left(\bar{b}_{L} \gamma^{\mu} s_{L}\right)\left(\bar{\mu}_{L} \gamma_{\mu} \mu_{L}\right)+\text { h.c. } \equiv \frac{1}{\Lambda_{K}^{2}}\left(\bar{b}_{L} \gamma^{\mu} s_{L}\right)\left(\bar{\mu}_{L} \gamma_{\mu} \mu_{L}\right)+\text { h.c. }
$$

As a consequence, the triplet leptoquark gives rise to the effective operator of the $(V-A)$ form for the quark current, that is, $C_{9}^{\mu, \mathrm{NP}}=-C_{10}^{\mu, \mathrm{NP}} \neq 0$, as favored by the $R_{K^{(*)}}$ anomalies, and the effective cutoff scale is to be $\Lambda_{K} \sim 30 \mathrm{TeV}$ [49]. The result is in contrast to the case for $Z^{\prime}$ models with family-dependent charges such as $Q^{\prime}=x\left(B_{3}-L_{3}\right)+y\left(L_{\mu}-L_{\tau}\right)$ with $x, y$ being arbitrary parameters where $C_{9}^{\mu, \mathrm{NP}} \neq 0$ and $C_{10}^{\mu, \mathrm{NP}}=0[60,61]$. Then, for $m_{\phi_{1}} \gtrsim 1 \mathrm{TeV}$, we need $\sqrt{\kappa_{32}^{*} \kappa_{22}} \gtrsim 0.03$. Therefore, we can combine scalar leptoquarks, $S_{1}$ and $S_{3}$, to explain $R_{D^{(*)}}$ and $R_{K^{(*)}}$ anomalies, respectively.

In the right plot of figure 1 , we depict the parameter space for $m_{S_{3}}$ and the effective leptoquark coupling, $\lambda_{\text {eff }}=\sqrt{\left|\kappa_{32}^{*} \kappa_{22}\right|}$, in which the $R_{K^{(*)}}$ anomalies can be explained within $2 \sigma(1 \sigma)$ errors in green(yellow) region from the conditions below eq. (2.3). 
Likewise as for the singlet scalar leptoquark, from the triplet leptoquark couplings necessary for $R_{K^{(*)}}$ anomalies,

$$
\begin{aligned}
\mathcal{L}_{S_{3}} \supset & -\kappa_{32}\left(\sqrt{2} \overline{\left(t^{C}\right)_{R}} \phi_{3} \nu_{\mu L}-\overline{\left(t^{C}\right)_{R}} \phi_{2} \mu_{L}-\overline{\left(b^{C}\right)_{R}} \phi_{2} \nu_{\mu L}-\sqrt{2} \overline{\left(b^{C}\right)_{R}} \phi_{1} \mu_{L}\right)+\text { h.c. } \\
& -\kappa_{22}\left(\sqrt{2} \overline{\left(c^{C}\right)_{R}} \phi_{3} \nu_{\mu L}-\overline{\left(c^{C}\right)_{R}} \phi_{2} \mu_{L}-\overline{\left(s^{C}\right)_{R}} \phi_{2} \nu_{\mu L}-\sqrt{2} \overline{\left(s^{C}\right)_{R}} \phi_{1} \mu_{L}\right)+\text { h.c. },
\end{aligned}
$$

the decay modes of the singlet scalar leptoquark are given by $\phi_{1} \rightarrow \bar{b} \bar{\mu}, \bar{s} \bar{\mu}, \phi_{2} \rightarrow$ $\bar{t} \bar{\mu}, \bar{c} \bar{\mu}, \bar{b} \bar{\nu}_{\mu}, \bar{s}_{\mu}$, and $\phi_{3} \rightarrow \bar{t} \bar{\nu}_{\mu}, \bar{c} \bar{\nu}_{\mu}$. As will be discussed in the next section, the bounds from $B \rightarrow K \nu \bar{\nu}$ could require $\kappa_{33}$ and $\kappa_{23}$ to be sizable. In this case, the decay modes containing $\bar{\tau}$ or $\bar{\nu}_{\tau}$ are relevant too. The decay branching ratios of the triplet leptoquark and the corresponding LHC bounds on the mass of triplet scalar leptoquark are also summarized in table 1.

\section{Constraints on leptoquarks}

We discuss the constraints on scalar leptoquark models, due to other rare meson decays, muon $(g-2)_{\mu}$, lepton flavor violation as well as the LHC searches. The constraints discussed in this section can give rise to important implications for the indirect signatures of DM annihilation into a leptoquark pair in the later discussion.

\subsection{Rare meson decays and mixing}

In leptoquark models explaining the B-meson anomalies, there is no $B-\bar{B}$ mixing at tree level, but instead it appears at one-loop level. Therefore, the resulting new contribution to the $B_{s}-\bar{B}_{s}$ mixing is about $1 \%$ level [26], which can be ignored.

Both singlet and triplet leptoquarks contribute to $B \rightarrow K^{(*)} \nu \bar{\nu}$ at tree level, so their couplings are severely constrained in this case [26, 28]. The effective Hamiltonian relevant for $\bar{b} \rightarrow \bar{s} \nu \bar{\nu}[62]$ is

$$
\mathcal{H}_{\bar{b} \rightarrow \bar{s} \nu \bar{\nu}}=-\frac{\sqrt{2} \alpha_{\mathrm{em}} G_{F}}{\pi} V_{t b} V_{t s}^{*} \sum_{l} C_{L}^{l}\left(\bar{b} \gamma^{\mu} P_{L} s\right)\left(\bar{\nu}_{l} \gamma_{\mu} P_{L} \nu_{l}\right)
$$

where $C_{L}^{l}=C_{L}^{\mathrm{SM}}+C_{\nu}^{l, \mathrm{NP}}$. Here, the SM contribution $C_{L}^{\mathrm{SM}}$ is given by $C_{L}^{\mathrm{SM}}=-X_{t} / s_{W}^{2}$ where $s_{W} \equiv \sin \theta_{W}$ and $X_{t}=1.469 \pm 0.017$. From the result in eq. (A.9), the scalar leptoquarks leads to additional contributions to the effective Hamiltonian for $B \rightarrow K \nu \bar{\nu}$ as

$$
C_{\nu}^{l, \mathrm{NP}}=-\left(\frac{\lambda_{3 i}^{*} \lambda_{2 j}}{2 m_{S_{1}}^{2}}+\frac{\kappa_{3 i}^{*} \kappa_{2 j}}{2 m_{\phi_{2}}^{2}}\right) \frac{\pi}{\sqrt{2} \alpha_{\mathrm{em}} G_{F} V_{t b} V_{t s}^{*}} .
$$

Therefore, the ratio of the branching ratios are given by

$$
\begin{aligned}
R_{K^{(*)} \nu} & \equiv \frac{B\left(B \rightarrow K^{(*)} \nu \bar{\nu}\right)}{\left.B\left(B \rightarrow K^{(*)} \nu \bar{\nu}\right)\right|_{\mathrm{SM}}} \\
& =\frac{2}{3}+\frac{1}{3} \frac{\left|C_{L}^{\mathrm{SM}}+C_{\nu}^{l, \mathrm{NP}}\right|^{2}}{\left|C_{L}^{\mathrm{SM}}\right|^{2}} .
\end{aligned}
$$


Comparing the experimental bounds on $B\left(B \rightarrow K^{(*)} \nu \bar{\nu}\right)$ [63] given by

$$
B(B \rightarrow K \nu \bar{\nu})<1.6 \times 10^{-5}, \quad B\left(B \rightarrow K^{*} \nu \bar{\nu}\right)<2.7 \times 10^{-5},
$$

to the SM values [64] given by

$$
\begin{gathered}
\left.B(B \rightarrow K \nu \bar{\nu})\right|_{\mathrm{SM}}=(3.98 \pm 0.43 \pm 0.19) \times 10^{-6}, \\
\left.B\left(B \rightarrow K^{*} \nu \bar{\nu}\right)\right|_{\mathrm{SM}}=(9.19 \pm 0.86 \pm 0.50) \times 10^{-6},
\end{gathered}
$$

and ignoring the imaginary part of $C_{\nu}^{l, \mathrm{NP}}$, we get the $R_{K^{*} \nu}$ bound as

$$
-10.1<\operatorname{Re}\left(C_{\nu}^{l, \mathrm{NP}}\right)<22.8 .
$$

Taking into account $\kappa_{32}$ and $\kappa_{22}$, which are necessary for $B \rightarrow K^{(*)} \mu^{+} \mu^{-}$, the triplet scalar leptoquark contributes only to $B \rightarrow K^{(*)} \nu_{\mu} \bar{\nu}_{\mu}$. In this case, as the triplet leptoquark contribution to $C_{\nu}^{\mu, \mathrm{NP}}$ is about the same as $C_{9}^{\mu, \mathrm{NP}}=-0.61$, it satisfies the $R_{K^{*} \nu}$ bound on its own easily.

On the other hand, the singlet leptoquark with nonzero $\lambda_{33}$ and $\lambda_{23}$, which are necessary for $B \rightarrow D^{(*)} \tau \bar{\nu}_{\tau}$, contribute significantly to $B \rightarrow K^{(*)} \nu_{\tau} \bar{\nu}_{\tau}$. Therefore, we need to cancel the singlet scalar leptoquark contributions to $B \rightarrow K^{(*)} \nu_{\tau} \bar{\nu}_{\tau}$, by imposing that

$$
\frac{\lambda_{33}^{*} \lambda_{23}}{2 m_{S_{1}}^{2}}+\frac{\kappa_{33}^{*} \kappa_{23}}{2 m_{\phi_{2}}^{2}} \approx 0 .
$$

Ignoring the mass splitting generated within the triplet scalar leptoquark due to potential higher dimensional operators after electroweak symmetry breaking, we get $m_{\phi_{1}}=$ $m_{\phi_{2}}=m_{\phi_{3}} \equiv m_{S_{3}}$. Then, in order to cancel the contributions to $B \rightarrow K^{(*)} \nu_{\tau} \bar{\nu}_{\tau}$ or $B \rightarrow K^{(*)} \nu_{\mu, \tau} \bar{\nu}_{\tau, \mu}$, the necessary conditions for the additional couplings are

$$
\begin{aligned}
& \left|\kappa_{33}^{*} \kappa_{23}\right| \approx\left|\lambda_{33}^{*} \lambda_{23}\right|\left(\frac{m_{S_{3}}^{2}}{m_{S_{1}}^{2}}\right), \\
& \left|\lambda_{32}^{*} \lambda_{23}\right| \approx\left|\kappa_{32}^{*} \kappa_{23}\right|\left(\frac{m_{S_{1}}^{2}}{m_{S_{3}}^{2}}\right) .
\end{aligned}
$$

Therefore, for $m_{S_{3}} \sim m_{S_{1}}$, the additional couplings for the triplet leptoquark, $\kappa_{23}$ and $\kappa_{33}$, must satisfy $\sqrt{\left|\kappa_{33}^{*} \kappa_{23}\right|} \approx \sqrt{\left|\lambda_{33}^{*} \lambda_{23}\right|} \gtrsim 0.4$, because $\sqrt{\lambda_{33}^{*} \lambda_{23}} \gtrsim 0.4$ to explain the $R_{D^{(*)}}$ anomalies. On the other hand, for $m_{S_{3}} \sim m_{S_{1}}$, the additional coupling for the singlet leptoquark, $\lambda_{32}$ must satisfy $\sqrt{\left|\lambda_{32}^{*} \lambda_{23}\right|} \approx \sqrt{\left|\kappa_{32}^{*} \kappa_{23}\right|}$, up to the conditions, $\sqrt{\lambda_{33}^{*} \lambda_{23}} \gtrsim 0.4$ and $\sqrt{\left|\kappa_{32}^{*} \kappa_{22}\right|} \gtrsim 0.03$, for explaining $R_{D^{(*)}}$ and $R_{K^{(*)}}$ anomalies, respectively. Then, it is easy to get a sizable $\lambda_{32}$ coupling in order to explain the deviation in $(g-2)_{\mu}$ as will be discussed later.

In summary, taking account of bounds from $B \rightarrow K^{(*)} \nu \bar{\nu}$, the necessary flavor structure for leptoquark couplings is given by the following,

$$
\lambda=\left(\begin{array}{ccc}
0 & 0 & 0 \\
0 & 0 & \lambda_{23} \\
0 & \lambda_{32} & \lambda_{33}
\end{array}\right), \quad \kappa=\left(\begin{array}{ccc}
0 & 0 & 0 \\
0 & \kappa_{22} & \kappa_{23} \\
0 & \kappa_{32} & \kappa_{33}
\end{array}\right) .
$$


If the extra couplings for $B \rightarrow K^{(*)} \nu \bar{\nu}$ are sizable, namely, $\lambda_{32} \sim \lambda_{23}, \lambda_{33}$ for the singlet leptoquark, and $\kappa_{23}, \kappa_{33} \gtrsim \kappa_{22}, \kappa_{32}$ for the triplet leptoquark, the decay branching ratios of leptoquarks are changed, so that the LHC searches for leptoquarks as well as the indirect searches for leptoquark portal dark matter will be affected. In particular, we will discuss the impact of extra couplings on the signatures of DM annihilations into a leptoquark pair in detail in the later section.

For the later discussion on $(g-2)_{\mu}$ in the next subsection, we illustrate some sets of consistent leptoquark couplings for $m_{S_{3}} \sim m_{S_{1}} \gtrsim 1 \mathrm{TeV}$. For $\lambda_{32}=\lambda_{33}=1$ and $\kappa_{23}=0.1$, we find that $\lambda_{23} \gtrsim 0.16, \kappa_{32} \sim \kappa_{33} \gtrsim 1.6$ and $\kappa_{22} \gtrsim 5.6 \times 10^{-4}$. In this case, we need a hierarchy of couplings, $\lambda_{32}=\lambda_{33} \gg \lambda_{23}$ and $\kappa_{32} \sim \kappa_{33} \gg \kappa_{23} \gg \kappa_{22}$. Instead, choosing $\lambda_{32}=\lambda_{33}=0.1$ and $\kappa_{23}=1$, we obtain that $\lambda_{23} \gtrsim 1.6, \kappa_{32} \sim 0.16, \kappa_{33} \gtrsim 0.16$ and $\kappa_{22} \gtrsim 5.6 \times 10^{-3}$. Then, we need a hierarchy of couplings, $\lambda_{23} \gg \lambda_{32}=\lambda_{33}$ and $\kappa_{23} \gg \kappa_{32} \sim \kappa_{33} \gg \kappa_{22}$.

\section{$4.2(g-2)_{\mu}$}

For the singlet scalar leptoquark, the relevant Yukawa couplings for $(g-2)_{\mu}$ with an additional Yukawa coupling, are given as follows,

$$
\mathcal{L}_{S_{1}} \supset-\lambda_{i j} \overline{\left(Q^{C}\right)_{R i}^{a}}\left(i \sigma^{2}\right)_{a b} S_{1} L_{j L}^{b}-\lambda_{i j}^{\prime} \overline{\left(u^{C}\right)_{L i}} S_{1} e_{j R}+\text { h.c. }
$$

Then, the chirality-enhanced effect from the top quark contributes most [26], as follows,

$$
a_{\mu}^{S_{1}}=\frac{m_{\mu}}{4 \pi^{2}} \operatorname{Re}\left[C_{R}^{22}\right]
$$

with

$$
C_{R}^{i j} \equiv-\frac{N_{c}}{12 m_{S_{1}}^{2}} m_{t} \lambda_{3 i} \lambda_{3 j}^{\prime *}\left(7+4 \log \left(\frac{m_{t}^{2}}{m_{S_{1}}^{2}}\right)\right) .
$$

The deviation of the anomalous magnetic moment of muon between experiment and SM values is given $[69,70]$ by

$$
\Delta a_{\mu}=a^{\exp }-a^{\mathrm{SM}}=288(80) \times 10^{-11},
$$

which is a $3.6 \sigma$ discrepancy from the SM [70]. We note that as discussed in eq. (4.9), the extra couplings for the triplet leptoquark, $\kappa_{23}$ and $\kappa_{33}$, allow for a sizable $\lambda_{32}$, leading to a large deviation in $(g-2)_{\mu}$ without a conflict to the bound from $B\left(B \rightarrow K^{(*)} \nu \bar{\nu}\right)$.

On the other hand, the additional coupling also contributes to the branching ratio of $\tau \rightarrow \mu \gamma$ as follows,

$$
\operatorname{BR}(\tau \rightarrow \mu \gamma)=\frac{\alpha m_{\tau}^{3}}{256 \pi^{4}} \tau_{\tau}\left(\left|C_{R}^{23}\right|^{2}+\left|C_{L}^{23}\right|^{2}\right)
$$

where $C_{L}^{i j}=C_{R}^{i j}\left(\lambda_{3 i} \rightarrow \lambda_{3 i}^{\prime}, \lambda_{3 j}^{\prime} \rightarrow \lambda_{3 j}\right)$ and the lifetime of tau is given by $\tau_{\tau}=(290.3 \pm$ $0.5) \times 10^{-15} \mathrm{~s}$ [70]. The current experimental bound is given [71] by

$$
\operatorname{BR}(\tau \rightarrow \mu \gamma)<4.4 \times 10^{-8}
$$

In figure 2, we show the parameter space for the singlet scalar leptoquark mass $m_{L Q}$ and the extra leptoquark coupling $\lambda_{32}^{\prime}$, where the $(g-2)_{\mu}$ anomaly can be explained, 

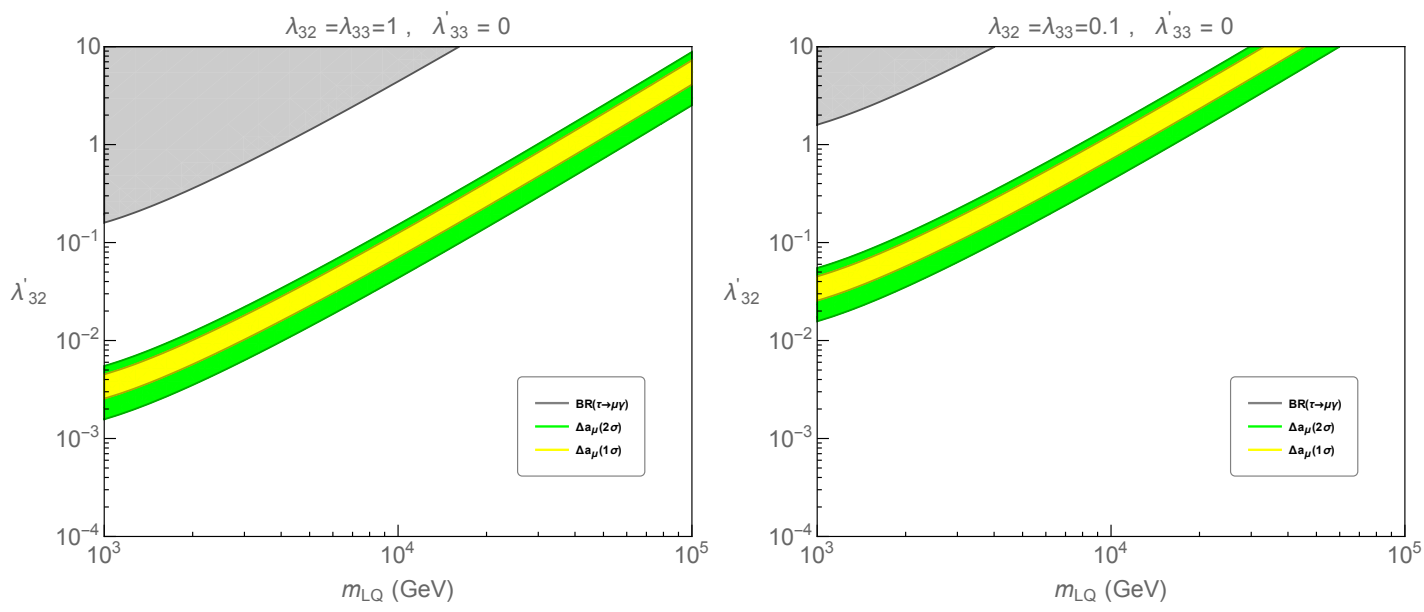

Figure 2. Parameter space for $m_{L Q}=m_{S_{1}}$ and $\lambda_{32}^{\prime}$ allowed by $(g-2)_{\mu}$, in green(yellow) region, at $2 \sigma(1 \sigma)$ level. The gray region is excluded by the bound on $\operatorname{BR}(\tau \rightarrow \mu \gamma)$. We have fixed $\lambda_{32}=\lambda_{33}=1(0.1)$ on left(right) plot and $\lambda_{33}^{\prime}=0$ in both plots.

in green(yellow) region at $2 \sigma(1 \sigma)$ level. The gray region is excluded by the bound on $B(\tau \rightarrow \mu \gamma)$. We have taken $\lambda_{32}=\lambda_{33}=1(0.1)$ on left(right) plot and $\lambda_{33}^{\prime}=0$. Therefore, for $m_{L Q} \lesssim 10-50 \mathrm{TeV}$ under perturbativity and leptoquark couplings less than unity, the $(g-2)_{\mu}$ anomaly can be explained in our model, being compatible with $B(\tau \rightarrow \mu \gamma)$.

\subsection{Leptoquark searches}

There are two main production channels for leptoquarks at the LHC, one is pair production via gluon fusion and the other is single production via gluon-quark fusion [25, 65-68].

In the case of $R_{K^{(*)}}$ anomalies, the triplet scalar leptoquark $\left(\phi_{1}\right)$ couples to $b / s, \mu$. The other components of the triplet leptoquark couple to $b / s, \nu_{\mu}$ and $t / c, \mu$ for $\phi_{2}$ and $t / c, \nu_{\mu}$ for $\phi_{3}$. On the other hand, in the case of $R_{D^{(*)}}$ anomalies, the singlet scalar leptoquark $\left(S_{1}\right)$ couples to $b / s, \nu_{\tau}$ and $t / c, \tau$. When the leptoquark pair production via gluon fusion is dominant, the current limits on leptoquark masses listed in table 1 apply. The current LHC bounds on leptoquarks depend on decay modes, but the leptoquark masses are constrained to be greater than about $1 \mathrm{TeV}$ in most cases.

When the Yukawa couplings, $\phi_{1}-b / s-\mu, S_{1}-b-\nu_{\tau}$ and $S_{1}-c-\tau$ couplings, present in models explaining the $B$-anomalies, are sizable, the leptoquarks can be singly produced by $b / s / c$ quark fusions with gluons. For instance, in the case of $\phi_{1}$, the relevant production/decay channels are $p p \rightarrow \phi_{1}^{*} \phi_{1}=b \bar{b}(s \bar{s}) \mu^{+} \mu^{-}$and $p p \rightarrow \phi_{1} \mu^{+} \rightarrow b(s) \mu^{+} \mu^{-}[25]$.

\section{Leptoquarks and scalar dark matter}

We introduce a scalar dark matter that have direct interactions to scalar leptoquarks and the SM Higgs doublet $H$ by quartic couplings. Thus, this is the minimal dark matter model without a need of extra mediator particle. In this section, we regard scalar leptoquarks as portals to scalar dark matter and discuss the impacts of leptoquarks on direct and indirect detection of dark matter as well as Higgs data. 

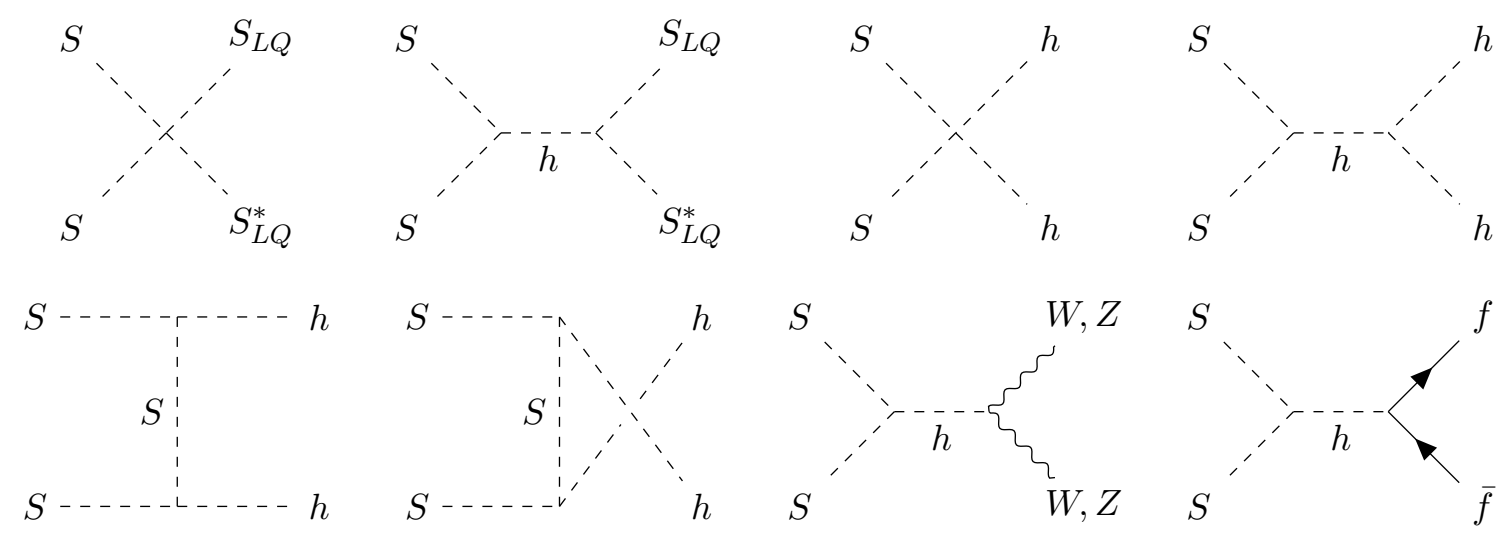

Figure 3. Feynman diagrams for annihilations of scalar dark matter at tree level.

We can also consider leptoquark-portal models for fermion or vector dark matter too. But, in this case, there is a need of mediator particles $[72,73]$ and/or non-renormalizable interactions $[74,75]$, leading to more parameters in the model, so this case is postponed to a future publication for comparison [76].

\subsection{Annihilation cross sections for scalar dark matter}

We consider a scalar leptoquark $S_{L Q}=S_{1,3}$ and a singlet real scalar dark matter $S$. Then, the most general renormalizable Lagrangian consistent with $S \rightarrow-S$ is

$$
\begin{aligned}
\mathcal{L}_{S}= & \left|D_{\mu} S_{L Q}\right|^{2}-m_{L Q}^{2}\left|S_{L Q}\right|^{2}+\frac{1}{2}\left(\partial_{\mu} S\right)^{2}-\frac{1}{2} m_{S}^{2} S^{2} \\
& -\frac{1}{4} \lambda_{1} S^{4}-\lambda_{2}\left|S_{L Q}\right|^{4}-\frac{1}{2} \lambda_{3} S^{2}\left|S_{L Q}\right|^{2}-\frac{1}{2} \lambda_{4} S^{2}|H|^{2}-\lambda_{5}|H|^{2}\left|S_{L Q}\right|^{2} .
\end{aligned}
$$

The above Lagrangian generalizes the Higgs portal interactions to those for leptoquarks.

After electroweak symmetry breaking with $H=(0, v+h)^{T} / \sqrt{2}$, the new interactions relevant for $S S \rightarrow S_{L Q} S_{L Q}^{*}, h h$ are

$$
\mathcal{L}_{\mathrm{S}, \mathrm{int}}=-\frac{1}{2} \lambda_{3} S^{2}\left|S_{L Q}\right|^{2}-\frac{1}{4} \lambda_{4} S^{2}\left(2 v h+h^{2}\right)-\frac{1}{2} \lambda_{5}\left|S_{\mathrm{LQ}}\right|^{2}\left(2 v h+h^{2}\right) .
$$

Scalar dark matter $S$ annihilates through three channels at tree level, $S S \rightarrow f \bar{f}$ with $f$ being the SM fermions, $S S \rightarrow h h$, with $h$ being the SM Higgs boson, $S S \rightarrow V V$ with $V$ being electroweak gauge bosons, and $S S \rightarrow S_{L Q} S_{L Q}^{*}$ for $m_{L Q}<m_{S}$. Feynman diagrams for the annihilation channels of scalar dark matter are shown in figure 3. Depending on the quartic couplings, a heavy scalar dark matter may annihilate into a pair of leptoquarks dominantly, leaving the signatures in both anti-proton and positron from cosmic rays, due to the decay products of leptoquarks, as will be discussed later.

For $m_{L Q}>m_{S}, S S \rightarrow S_{L Q} S_{L Q}^{*}$ channels are kinematically closed, so instead leptoquark loops make corrections to $S S \rightarrow V V$ with $V$ being electroweak gauge bosons and contribute to new annihilations such as $S S \rightarrow g g, Z \gamma, \gamma \gamma$. In this case, depending on the relative contributions of $S S \rightarrow f \bar{f}, h h, V V$ channels, the loop-induced annihilation channels can be relevant. 
We obtain the effective interactions between scalar dark matter and the SM gauge bosons due to leptoquarks with $m_{L Q}>m_{S}$, as follows,

$$
\mathcal{L}_{S, \text { eff }}=D_{3} S^{2} G_{\mu \nu} G^{\mu \nu}+D_{2} S^{2} W_{\mu \nu} W^{\mu \nu}+D_{1} S^{2} F_{Y \mu \nu} F^{Y \mu \nu}
$$

The details on the above effective interactions are given in appendix B. Then, in the basis of mass eigenstates, the above effective interactions become

$$
\begin{aligned}
\mathcal{L}_{S, \mathrm{eff}}= & D_{g g} S^{2} G_{\mu \nu} G^{\mu \nu}+D_{W W} S^{2} W_{\mu \nu}^{+} W^{-\mu \nu}+D_{Z Z} S^{2} Z_{\mu \nu} Z^{\mu \nu} \\
& +D_{Z \gamma} S^{2} Z_{\mu \nu} F^{\mu \nu}+D_{\gamma \gamma} S^{2} F_{\mu \nu} F^{\mu \nu}
\end{aligned}
$$

where

$$
\begin{aligned}
D_{g g} & =D_{3} \\
D_{W W} & =2 D_{2} \\
D_{Z Z} & =D_{1} \sin ^{2} \theta_{W}+D_{2} \cos ^{2} \theta_{W} \\
D_{Z \gamma} & =\left(D_{2}-D_{1}\right) \sin \left(2 \theta_{W}\right) \\
D_{\gamma \gamma} & =D_{1} \cos ^{2} \theta_{W}+D_{2} \sin ^{2} \theta_{W}
\end{aligned}
$$

First, the tree-level annihilation cross sections are

$$
\begin{aligned}
\left(\sigma v_{\mathrm{rel}}\right)_{S S \rightarrow S_{L Q} S_{L Q}^{*}} & =\frac{N_{c} N_{L Q}}{32 \pi m_{S}^{2}} \sqrt{1-\frac{m_{L Q}^{2}}{m_{S}^{2}}}\left(\lambda_{3}+\frac{\lambda_{4} \lambda_{5} v^{2}}{4 m_{S}^{2}-m_{h}^{2}}\right)^{2} \\
\left(\sigma v_{\mathrm{rel}}\right)_{S S \rightarrow h h} & =\frac{\lambda_{4}^{2}}{64 \pi m_{S}^{2}} \sqrt{1-\frac{m_{h}^{2}}{m_{S}^{2}}}\left(1+\frac{3 m_{h}^{2}}{4 m_{S}^{2}-m_{h}^{2}}-\frac{2 \lambda_{4} v^{2}}{2 m_{S}^{2}-m_{h}^{2}}\right)^{2} \\
\left(\sigma v_{\mathrm{rel}}\right)_{S S \rightarrow f \bar{f}} & =\frac{N_{c} \lambda_{4}^{2}}{4 \pi} \frac{m_{f}^{2}}{\left(4 m_{S}^{2}-m_{h}^{2}\right)^{2}}\left(1-\frac{m_{f}^{2}}{m_{S}^{2}}\right)^{3 / 2}
\end{aligned}
$$

with $f$ being all the SM fermions satisfying $m_{f}<m_{S}$. Here, we note that $N_{c}=3$ is the number of colors and $N_{L Q}=1,3$ for $S_{L Q}=S_{1}, S_{3}$, respectively.

On the other hand, for $m_{L Q}>m_{S}$, instead of $S S \rightarrow S_{L Q} S_{L Q}^{*}$, we need to consider the loop-induced annihilation cross sections [77-81] for $S S \rightarrow g g, \gamma \gamma, Z \gamma$, as follows,

$$
\begin{aligned}
\left(\sigma v_{\mathrm{rel}}\right)_{S S \rightarrow g g} & =\frac{64 D_{g g}^{2} m_{S}^{2}}{\pi}, \\
\left(\sigma v_{\mathrm{rel}}\right)_{S S \rightarrow \gamma \gamma} & =\frac{8 D_{\gamma \gamma}^{2} m_{S}^{2}}{\pi}, \\
\left(\sigma v_{\mathrm{rel}}\right)_{S S \rightarrow Z \gamma} & =\frac{4 D_{Z \gamma}^{2} m_{S}^{2}}{\pi}\left(1-\frac{m_{Z}^{2}}{4 m_{S}^{2}}\right)^{3} .
\end{aligned}
$$

Adding loop corrections of leptoquarks to tree-level contributions coming from the Higgs portal coupling $\lambda_{4}$, we also obtain the annihilation cross sections for $S S \rightarrow W W, Z Z$, 

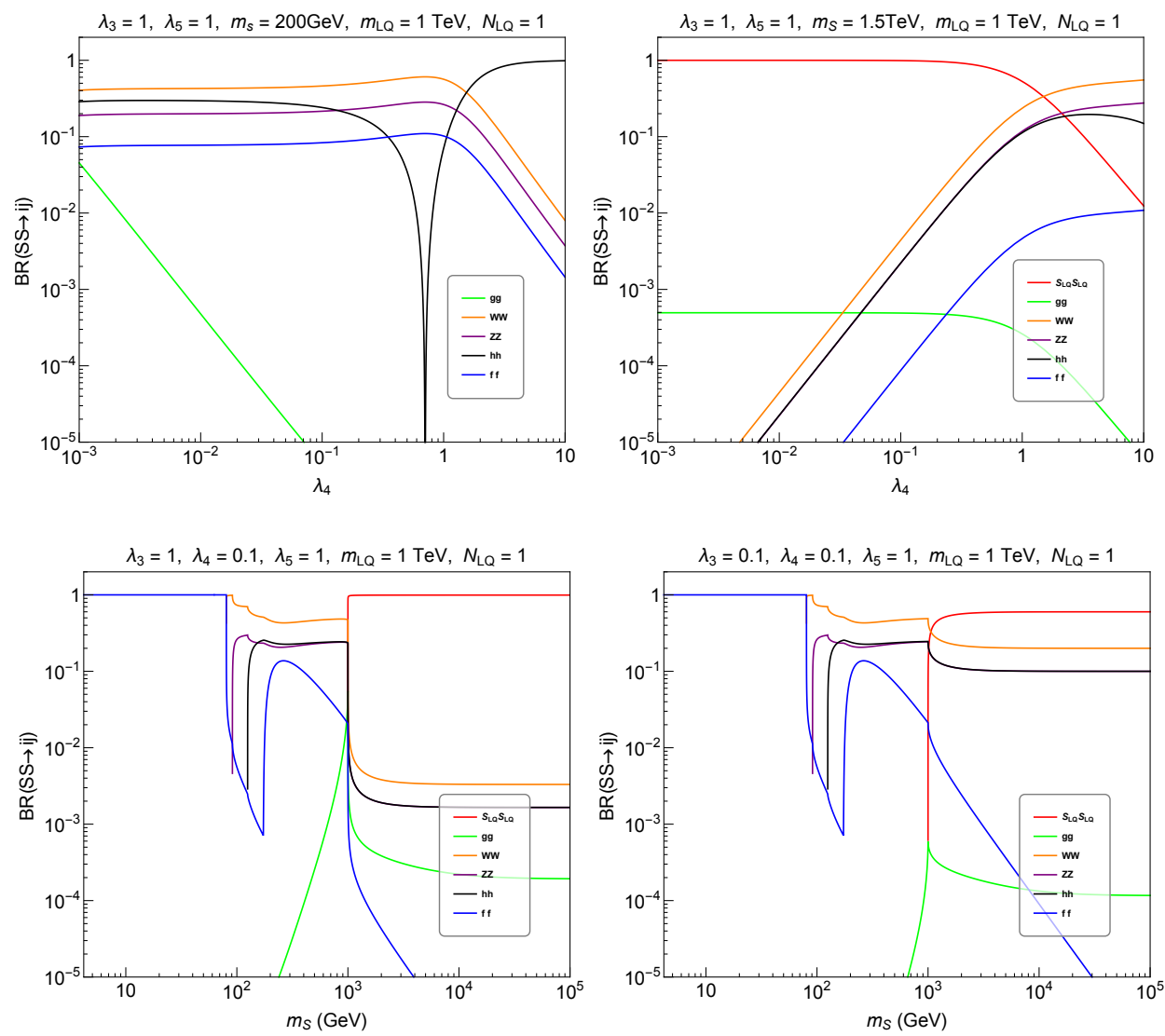

Figure 4. Branching ratios of annihilation cross sections for dark matter as a function of $\lambda_{4}$ (upper panel) or $m_{S}$ (lower panel), in models with singlet scalar leptoquark. Branching ratios for $W W$ (orange), $Z Z$ (purple), $g g$ (green), $h h$ (black), $f \bar{f}$ (blue), and $S_{L Q} S_{L Q}^{*}$ (red) channels are shown.

respectively,

$$
\begin{aligned}
\left(\sigma v_{\mathrm{rel}}\right)_{S S \rightarrow W W}= & {\left[\frac{\lambda_{4}^{2} m_{S}^{2}}{2 \pi\left(m_{h}^{2}-4 m_{S}^{2}\right)^{2}}\left(1-\frac{m_{W}^{2}}{m_{S}^{2}}+\frac{3 m_{W}^{4}}{4 m_{S}^{4}}\right)+\frac{4\left|D_{W W}\right|^{2} m_{S}^{2}}{\pi}\left(1-\frac{m_{W}^{2}}{m_{S}^{2}}+\frac{3 m_{W}^{4}}{8 m_{S}^{4}}\right)\right.} \\
& \left.+\frac{3 \lambda_{4} \operatorname{Re}\left[D_{W W}\right] m_{W}^{2}}{2 \pi\left(m_{h}^{2}-4 m_{S}^{2}\right)}\left(2-\frac{m_{W}^{2}}{m_{S}^{2}}\right)\right] \sqrt{1-\frac{m_{W}^{2}}{m_{S}^{2}}}, \\
\left(\sigma v_{\mathrm{rel}}\right)_{S S \rightarrow Z Z}= & {\left[\frac{\lambda_{4}^{2} m_{S}^{2}}{4 \pi\left(m_{h}^{2}-4 m_{S}^{2}\right)^{2}}\left(1-\frac{m_{Z}^{2}}{m_{S}^{2}}+\frac{3 m_{Z}^{4}}{4 m_{S}^{4}}\right)+\frac{8\left|D_{Z Z}\right|^{2} m_{S}^{2}}{\pi}\left(1-\frac{m_{Z}^{2}}{m_{S}^{2}}+\frac{3 m_{Z}^{4}}{8 m_{S}^{4}}\right)\right.} \\
& \left.+\frac{3 \lambda_{4} \operatorname{Re}\left[D_{Z Z}\right] m_{Z}^{2}}{2 \pi\left(m_{h}^{2}-4 m_{S}^{2}\right)}\left(2-\frac{m_{Z}^{2}}{m_{S}^{2}}\right)\right] \sqrt{1-\frac{m_{Z}^{2}}{m_{S}^{2}}} .
\end{aligned}
$$

In figures 4 and 5, we show the branching ratios of annihilation cross sections of dark matter, $\operatorname{BR}(S S \rightarrow i j)$, as a function of $\lambda_{4}$ in the upper panel and $m_{S}$ in the lower panel, for singlet and triplet scalar leptoquarks, respectively. 

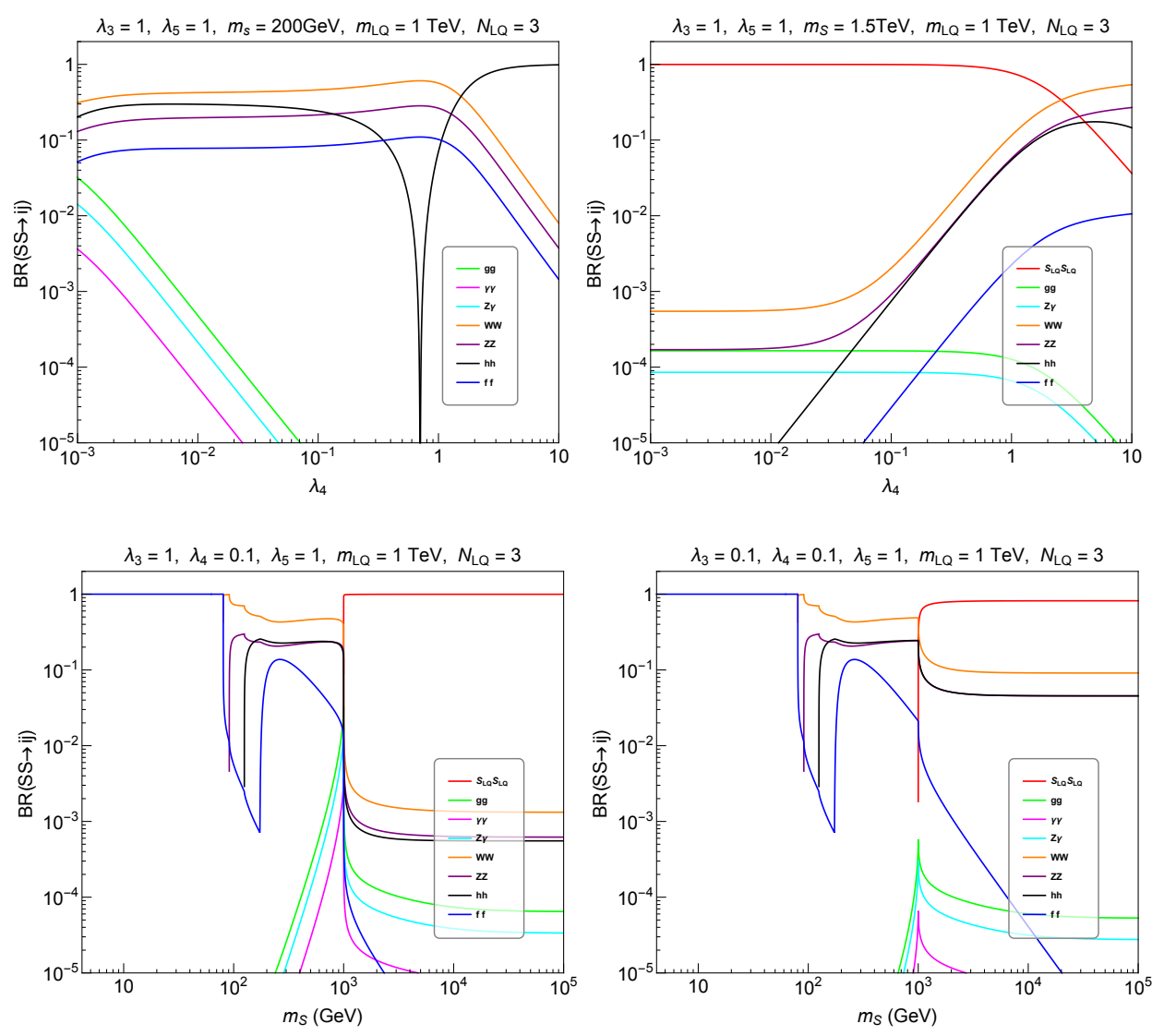

Figure 5. The same as in figure 4, but in models with triplet scalar leptoquark.

For light dark matter with $m_{S}<m_{L Q}$, we find that the tree-level annihilation processes such as $W W, h h, f \bar{f}, Z Z$ are dominant and the loop-induced processes due to leptoquarks are suppressed, except the $g g$ channel, which can be as large as $1-10 \%$ of the total annihilation cross section, depending on whether the scalar leptoquark is singlet or triplet. In the case of triplet scalar leptoquark, the $Z \gamma, \gamma \gamma$ channels can be as large as $1 \%$ or $0.1 \%$ of the total annihilation cross section, so they could be probed by Fermi-LAT [83] or HESS $[84,85]$ line searches. On the other hand, for heavy dark matter with $m_{S}>m_{L Q}$, the $S_{L Q} S_{L Q}^{*}$ channel becomes dominant while the other tree-level processes are negligible as far as $\left|\lambda_{3}\right| \gtrsim\left|\lambda_{4}\right|$.

\subsection{Direct detection bounds}

For scalar dark matter, the effective DM-quark interaction is induced due to the SM Higgs exchange at tree level, as follows,

$$
\mathcal{L}_{\mathrm{eff}, S q \bar{q}}=\frac{\lambda_{4} m_{q}}{m_{h}^{2}} S^{2} \bar{q} q
$$

Moreover, taking a small momentum transfer for the DM-nucleon scattering in eq. (B.2), the effective interactions between scalar dark matter and gluons, generated by loop correc- 
tions with leptoquarks, become

$$
\mathcal{L}_{\mathrm{eff}, S g g}=\frac{\alpha_{S} \lambda_{4}}{96 \pi m_{\mathrm{LQ}}^{2}} l_{3}\left(S_{L Q}\right) S^{2} G_{\mu \nu} G^{\mu \nu}
$$

where $l_{3}\left(S_{L Q}\right)$ is the Dynkin index of a leptoquark $S_{L Q}$ under $\mathrm{SU}(3)_{C}$. Then, the spinindependent cross section for DM-nucleon elastic scattering is given by

$$
\sigma_{S-N}=\frac{\mu_{N}^{2}}{\pi m_{S}^{2} A^{2}}\left(Z f_{p}+(A-Z) f_{n}\right)^{2}
$$

where $Z, A-Z$ are the numbers of protons and neutrons in the detector nucleus, $\mu_{N}=$ $m_{N} m_{S} /\left(m_{N}+m_{S}\right)$ is the reduced mass of DM-nucleon system, and

$$
f_{p, n}=\frac{\lambda_{4} m_{p, n}}{m_{h}^{2}}\left(\sum_{q=u, d, s} f_{T q}^{p, n}+\frac{2}{9} f_{T G}^{p, n}\right)-\frac{\lambda_{3} m_{p, n}}{108 m_{L Q}^{2}} l_{3}\left(S_{L Q}\right) f_{T G}^{p, n}
$$

with $f_{T G}^{p, n}=1-\sum_{q=u, d, s} f_{T q}^{p, n}$. Here, the mass fractions are $f_{T_{u}}^{p}=0.023, f_{T_{d}}^{p}=0.032$ and $f_{T_{s}}^{p}=0.020$ for a proton and $f_{T_{u}}^{n}=0.017, f_{T_{d}}^{n}=0.041$ and $f_{T_{s}}^{n}=0.020$ for a neutron [82]. Therefore, the quartic coupling $\lambda_{4}$ between scalar dark matter and SM Higgs is strongly constrained by direct detection experiments such as XENON1T [17, 18]. Consequently, tree-level annihilations of scalar dark matter into $h h, f \bar{f}, W W, Z Z$ are constrained, while the leptoquark-induced annihilations at tree or loop levels can be relevant.

In figure 6, we show the DM relic density as a function of DM mass in red solid(dashed) lines for triplet(singlet) scalar leptoquarks. We also show the DM-nucleon scattering cross section in blue lines as can be read from the right vertical axis, and the XENON1T bound in purple dot-dashed lines. We find that the extra annihilation of dark matter into a pair of leptoquarks opens a new parameter space at $m_{S}>m_{L Q}$ due to a sizable leptoquark portal coupling, $\lambda_{3}$, avoiding the direct detection bound from XENON1T.

\subsection{Indirect detection bounds}

For relatively light scalar dark matter with $m_{S}<m_{L Q}$, the DM annihilation cross sections into $h h, W W, Z Z, t \bar{t}, b \bar{b}$ are dominant. In this case, Fermi-LAT dwarf galaxies [86] and HESS gamma-rays [87] and AMS-02 antiprotons [88, 89] can constrain the model.

In figure 7 , we depict the parameter space in $\lambda_{4}$ vs $m_{S}$ in black and red solid lines, satisfying the correct relic density for models with singlet and triplet scalar leptoquarks, respectively. In the same plots, we superimpose the indirect detection bounds on the DM annihilations into a $W W$ pair from Fermi-LAT and HESS gamma-ray and AMS-02 antiproton searches, and include the direct detection bounds from XENON1T. Moreover, the region with $m_{S}<m_{h} / 2$ can be also constrained by Higgs data such as Higgs invisible decay and the signal strength for $g g \rightarrow h \rightarrow \gamma \gamma$, as will be discussed in the next subsection.

As a result, the Higgs data as well as indirect detection constrains the region with light and weak-scale dark matter, but the XENON1T experiment constrains most, ruling out most of the DM masses below $m_{S}=1 \mathrm{TeV}$, except the resonance region near $m_{S}=m_{h} / 2$. However, we find that the correct relic density can be obtained for a small value of $\lambda_{4}$ 

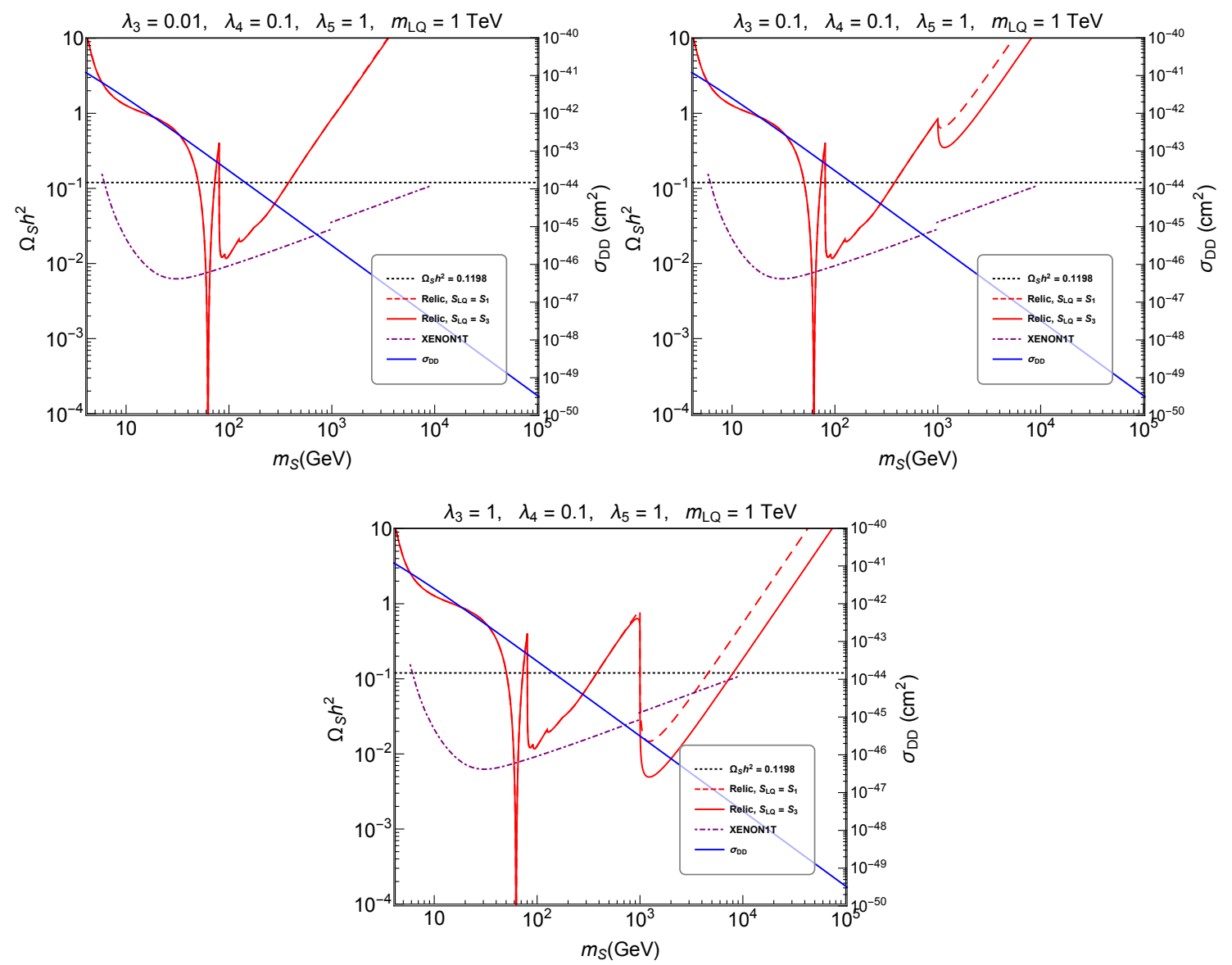

Figure 6. Dark matter relic density as a function of $m_{S}$ in red solid (dashed) lines for triplet (singlet) scalar leptoquarks. DM-nucleon scattering cross section and XENON1T bound are shown in blue line and purple dot-dashed line, respectively. $\lambda_{3}=0.01,0.1,1$ are taken from the top left plot clockwise, and $\lambda_{4}=0.1, \lambda_{5}=1$ and $m_{L Q}=1 \mathrm{TeV}$ are taken for all plots.

due to the contribution of DM annihilation channels into a leptoquark pair with a sizable leptoquark-portal coupling $\lambda_{3}$ for $m_{S}>m_{L Q}$. Therefore, there is a wide parameter space above $m_{S}=1 \mathrm{TeV}$ that is consistent with the XENON1T bound.

We remark the indirect signatures of dark matter and the leptoquark decays in the case of heavy scalar dark matter. For $m_{S}>m_{L Q}$, dark matter can annihilate into a pair of leptoquarks, each of which decays into a pair of quark and lepton in cascade. In the case with leptoquark couplings to explain the $B$-meson anomalies, the branching ratios of final products of DM annihilations are shown in table 2, depending on the decay branching ratios of leptoquarks. In the case where the extra leptoquark couplings, $\lambda_{32}, \kappa_{23}$ and $\kappa_{33}$, introduced for accommodating $B \rightarrow K^{(*)} \bar{\nu} \nu$ bounds and/or the $(g-2)_{\mu}$ excess, are dominant, as discussed in section 4.1, we also show the corresponding branching ratios of final products of DM annihilations in table 3 .

In particular, for $m_{S} \gtrsim m_{L Q}$, a leptoquark pair is produced with almost zero velocities, so each leptoquark decays into a pair of quark and lepton such as $\bar{q} \bar{l}$ or $q^{\prime} l^{\prime}$, back-to-back. 

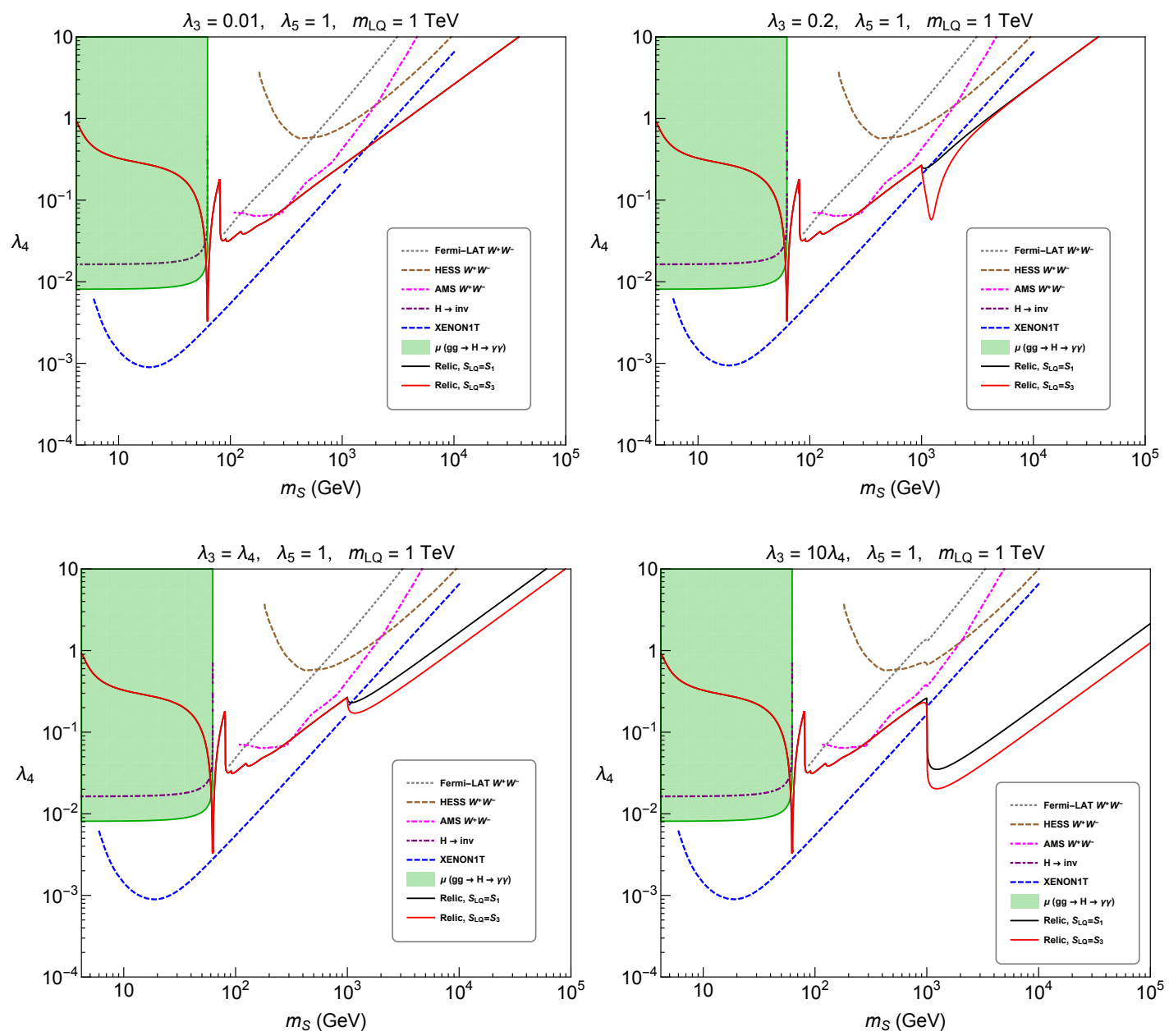

Figure 7. Relic density for scalar dark matter and various bounds in the parameter space, $\lambda_{4}$ vs $m_{S}$. The correct relic density can be obtained along the black and red solid lines, for models with singlet and triplet scalar leptoquarks, respectively. XENON1T bounds are shown in blue dashed lines. Indirect detection constraints from gamma-ray searches in Fermi-LAT (gray dotted) and HESS (brown dashed), and antiproton search in AMS-02 (pink dot-dashed) are overlaid. The bound from Higgs invisible decay is shown in purple dot-dashed line and the green regions are excluded by visible decays such as the Higgs diphoton signal strength.

In this case, a pair of two quarks $\left(q^{\prime} \bar{q}\right)$ or a pair of leptons $\left(l^{\prime} \bar{l}\right)$ carry about the energy of DM mass, so we take them as if they are produced from the direct annihilations of dark matter with mass $m_{S} / 2$ and impose the indirect detection bounds on the annihilation cross section. But, if $m_{S} \gg m_{L Q}$, leptoquarks produced from the DM annihilations are boosted so the full energy spectra for quarks or leptons carry the energy spectra of wide box rather than a monochromatic energy. In this case, we need to take more care before imposing the indirect detection bounds. Henceforth, ignoring the boost effects of leptoquarks, in particular, for $m_{S} \gtrsim m_{L Q}$, we discuss the indirect detection bounds for the direct annihilations of dark matter to cascade annihilations.

First, we consider the case in table 2 with leptoquark couplings necessary to explain the $B$-meson anomalies. In this case, for a singlet leptoquark with $\lambda_{33} \gg \lambda_{23}$ or $\beta \approx 1$, 


\begin{tabular}{|c|c|c|c|}
\hline LQs & BRs & BRs & BRs \\
\hline$S_{1} S_{1}^{*}$ & $\mathrm{~B}\left(\left|\bar{t} \bar{\tau}+b \nu_{\tau}\right|^{2}\right)$ & $\mathrm{B}\left(\left|\bar{c} \bar{\tau}+s \nu_{\tau}\right|^{2}\right)$ & $\mathrm{B}\left(\left(\bar{t} \bar{\tau}+b \nu_{\tau}\right)^{*}\left(\bar{c} \bar{\tau}+s \nu_{\tau}\right)+\right.$ h.c. $)$ \\
& $=\beta^{2}$ & $=(1-\beta)^{2}$ & $=2 \beta(1-\beta)$ \\
\hline$\phi_{1} \phi_{1}^{*}$ & $\mathrm{~B}(\bar{b} b \bar{\mu} \mu)$ & $\mathrm{B}(\bar{s} s \bar{\mu} \mu)$ & $\mathrm{B}(\bar{b} s \bar{\mu} \mu+$ h.c. $)$ \\
& $=\gamma^{2}$ & $=(1-\gamma)^{2}$ & $=2 \gamma(1-\gamma)$ \\
\hline$\phi_{2} \phi_{2}^{*}$ & $\mathrm{~B}\left(\left|\bar{t} \bar{\mu}+b \nu_{\mu}\right|^{2}\right)$ & $\mathrm{B}\left(\left|\bar{c} \bar{\mu}+s \nu_{\mu}\right|^{2}\right)$ & $\mathrm{B}\left(\left(\bar{t} \bar{\mu}+b \nu_{\mu}\right)^{*}\left(\bar{c} \bar{\mu}+s \nu_{\mu}\right)+\right.$ h.c. $)$ \\
& $=\gamma^{2}$ & $=(1-\gamma)^{2}$ & $=2 \gamma(1-\gamma)$ \\
\hline$\phi_{3} \phi_{3}^{*}$ & $\mathrm{~B}\left(\bar{t} t \bar{\nu}_{\mu} \nu_{\mu}\right)$ & $\mathrm{B}\left(\bar{c} c \bar{\nu}_{\mu} \nu_{\mu}\right)$ & $\mathrm{B}\left(\bar{t} c \bar{\nu}_{\mu} \nu_{\mu}+\right.$ h.c. $)$ \\
& $=\gamma^{2}$ & $=(1-\gamma)^{2}$ & $=2 \gamma(1-\gamma)$ \\
\hline
\end{tabular}

Table 2. Branching ratios of products of DM annihilations into leptoquarks. Here, $\beta \equiv \lambda_{33}^{2} /\left(\lambda_{33}^{2}+\right.$ $\left.\lambda_{23}^{2}\right)$ and $\gamma \equiv \kappa_{32}^{2} /\left(\kappa_{32}^{2}+\kappa_{22}^{2}\right)$.

we get the branching ratios of products of $\mathrm{DM}$ annihilations into leptoquarks as $\mathrm{B}(\bar{t} t \bar{\tau} \tau)$ : $\mathrm{B}\left(\bar{b} b \bar{\nu}_{\tau} \nu_{\tau}\right): \mathrm{B}\left(\bar{t} b \bar{\tau} \nu_{\tau}+\right.$ h.c. $)=\frac{1}{2}: \frac{1}{2}: 1$. Then, we can impose the Fermi-LAT diffuse gamma-ray constraints from $\bar{b} b$ and $\bar{\tau} \tau$ [86] on $\frac{1}{4}\langle\sigma v\rangle_{S S \rightarrow S_{L Q} S_{L Q}^{*}}$. Similarly, for a triplet leptoquark with $\kappa_{32} \gg \kappa_{22}$ or $\gamma \approx 1$, we get $\mathrm{B}(\bar{b} b \bar{\mu} \mu): \mathrm{B}(\bar{t} t \bar{\mu} \mu): \mathrm{B}\left(\bar{b} b \bar{\nu}_{\mu} \nu_{\mu}\right): \mathrm{B}\left(\bar{t} b \bar{\mu} \nu_{\mu}+\right.$ h.c.) : $\mathrm{B}\left(\bar{t} t \bar{\nu}_{\mu} \nu_{\mu}\right)=1: \frac{1}{4}: \frac{1}{4}: \frac{1}{2}: 1$. In this case, we can impose the Fermi-LAT bounds for $\bar{b} b$ and $\bar{\mu} \mu[86]$ on $\frac{5}{12}\langle\sigma v\rangle_{S S \rightarrow S_{L Q} S_{L Q}^{*}}$ too. In general, positron, anti-proton and gamma-ray constraints are equally relevant for leptoquark-portal dark matter.

In figure 8 , in the parameter space in $\lambda_{4}$ vs $m_{S}$, in addition to the correct relic density conditions for models with singlet and triplet scalar leptoquarks, respectively, in black and red solid lines and the direct detection bounds from XENON1T, we superimpose the Fermi-LAT constraints from $b \bar{b}$ and $\tau \bar{\tau}$ on the products of DM cascade annihilations into a leptoquark pair. Here, we assume that each leptoquark decays into a pair of quark and lepton, according to table 2 with $\beta \approx 1$ and $\gamma \approx 1$. Then, as explained in the caption of figure 8, the resulting Fermi-LAT bounds are shown to constrain the parameter space as strong as or stronger than the XENON1T bounds, depending on the value of leptoquarkportal coupling $\lambda_{3}$.

Next, we consider the case in table 3 where the extra leptoquark couplings introduced for accommodating $B \rightarrow K^{(*)} \bar{\nu} \nu$ bounds and/or the $(g-2)_{\mu}$ excess are dominant. In this case, for a singlet leptoquark, we get the branching ratios of products of DM annihilations into leptoquarks as $\mathrm{B}(\bar{t} t \bar{\mu} \mu): \mathrm{B}\left(\bar{b} b \bar{\nu}_{\mu} \nu_{\mu}\right): \mathrm{B}\left(\bar{t} b \bar{\mu} \nu_{\mu}+\right.$ h.c. $)=\frac{1}{2}: \frac{1}{2}: 1$. Then, we can impose the Fermi-LAT diffuse gamma-ray constraints from $\bar{b} b$ and $\bar{\mu} \mu[86]$ on $\frac{1}{4}\langle\sigma v\rangle_{S S \rightarrow S_{L Q} S_{L Q}^{*}}$ as for the case in table 2. Similarly, for a triplet leptoquark with $\kappa_{33} \gg \kappa_{23}$ as in the first benchmark point discussed in the last paragraph in section 4.1 or $\gamma^{\prime} \approx 1$, we get $\mathrm{B}(\bar{b} b \bar{\tau} \tau): \mathrm{B}(\bar{t} t \bar{\tau} \tau): \mathrm{B}\left(\bar{b} b \bar{\nu}_{\tau} \nu_{\tau}\right): \mathrm{B}\left(\bar{t} b \bar{\tau} \nu_{\tau}+\right.$ h.c. $): \mathrm{B}\left(\bar{t}_{t} \bar{\nu}_{\tau} \nu_{\tau}\right)=1: \frac{1}{4}: \frac{1}{4}: \frac{1}{2}: 1$. In this case, the similar Fermi-LAT bounds for $\bar{b} b$ and $\bar{\tau} \tau$ [86] can be also imposed on $\frac{5}{12}\langle\sigma v\rangle_{S S \rightarrow S_{L Q} S_{L Q}^{*}}$ as for the case in table 2 . 

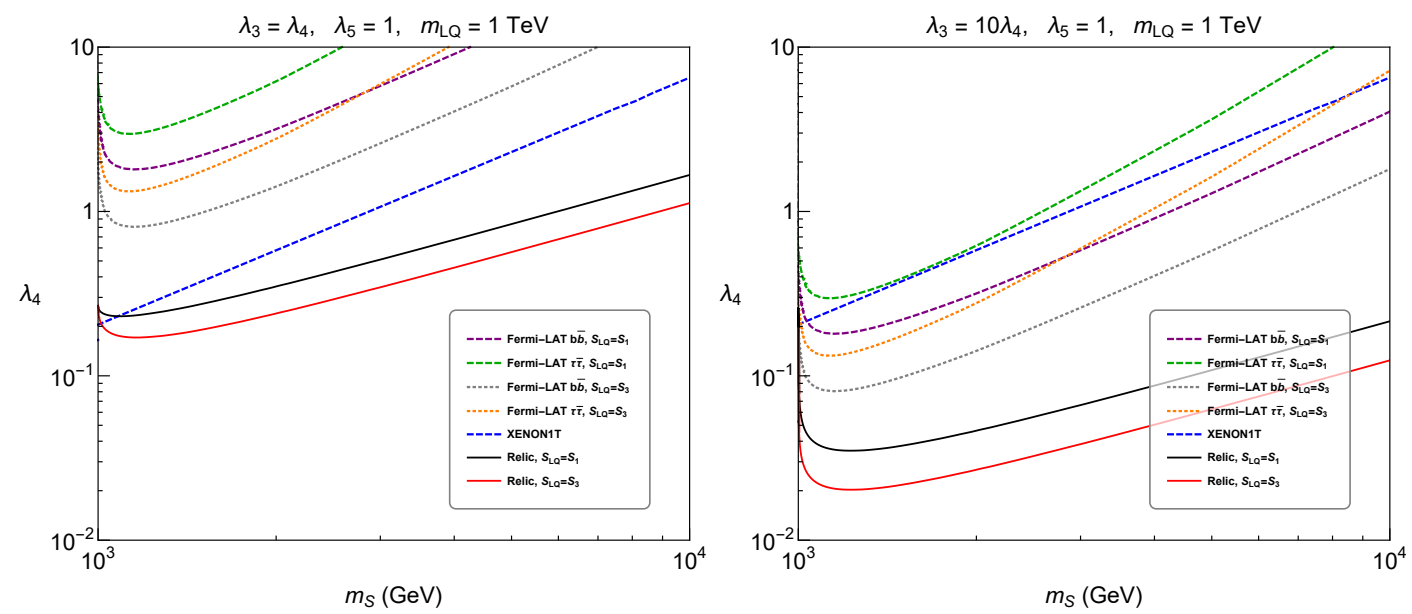

Figure 8. Relic density for scalar dark matter and various bounds in the parameter space, $\lambda_{4}$ vs $m_{S}$. The correct relic density can be obtained along the black and red solid lines, for models with singlet and triplet scalar leptoquarks, respectively. XENON1T bounds are shown in blue dashed lines. Fermi-LAT gamma-ray constraints on $b \bar{b}$ and $\tau \bar{\tau}$ coming from cascade annihilations are also shown in purple dashed (gray dotted) and green dashed (orange dotted) lines for singlet (triplet) leptoquarks.

\begin{tabular}{|c|c|c|c|}
\hline LQs & BRs & BRs & BRs \\
\hline$S_{1} S_{1}^{*}$ & $\mathrm{~B}(\bar{t} t \bar{\mu} \mu)=\frac{1}{4}$ & $\mathrm{~B}\left(\bar{b} b \bar{\nu}_{\mu} \nu_{\mu}\right)=\frac{1}{4}$ & $\mathrm{~B}\left(\bar{t} b \bar{\mu} \nu_{\mu}+\right.$ h.c. $)=\frac{1}{2}$ \\
\hline$\phi_{1} \phi_{1}^{*}$ & $\mathrm{~B}(\bar{b} b \bar{\tau} \tau)=\gamma^{\prime 2}$ & $\mathrm{~B}(\bar{s} s \bar{\tau} \tau)=\left(1-\gamma^{\prime}\right)^{2}$ & $\mathrm{~B}(\bar{b} s \bar{\tau} \tau+$ h.c. $)=2 \gamma^{\prime}\left(1-\gamma^{\prime}\right)$ \\
\hline$\phi_{2} \phi_{2}^{*}$ & $\mathrm{~B}\left(\left|\bar{t} \bar{\tau}+b \nu_{\tau}\right|^{2}\right)$ & $\mathrm{B}\left(\left|\bar{c} \bar{\tau}+s \nu_{\tau}\right|^{2}\right)$ & $\mathrm{B}\left(\left(\bar{t} \bar{\tau}+b \nu_{\tau}\right)^{*}\left(\bar{c} \bar{\tau}+s \nu_{\tau}\right)+\right.$ h.c. $)$ \\
& $=\gamma^{\prime 2}$ & $=\left(1-\gamma^{\prime}\right)^{2}$ & $=2 \gamma^{\prime}\left(1-\gamma^{\prime}\right)$ \\
\hline$\phi_{3} \phi_{3}^{*}$ & $\mathrm{~B}\left(\bar{t} t \bar{\nu}_{\tau} \nu_{\tau}\right)=\gamma^{\prime 2}$ & $\mathrm{~B}\left(\bar{c} c \bar{\nu}_{\tau} \nu_{\tau}\right)=\left(1-\gamma^{\prime}\right)^{2}$ & $\mathrm{~B}\left(\bar{t}_{c} \bar{\nu}_{\tau} \nu_{\tau}+\right.$ h.c. $)=2 \gamma^{\prime}\left(1-\gamma^{\prime}\right)$ \\
\hline
\end{tabular}

Table 3. Branching ratios of products of DM annihilations into leptoquarks, for the dominance of the extra couplings, $\lambda_{32}, \kappa_{23}$ and $\kappa_{33}$. Here, $\gamma^{\prime} \equiv \kappa_{33}^{2} /\left(\kappa_{23}^{2}+\kappa_{33}^{2}\right)$.

In summary, the leptoquark-portal couplings lead to potentially distinct signatures with quarks and leptons mixed from the cascade annihilations of dark matter, as compared to the case with direct annihilations into a quark pair or a lepton pair. Our lepto-quark portal scenario is different from the Higgs portal scenario with additional $\mathrm{SU}(2)_{L}$ singlet or triplet scalars, because the final states in the cascade DM annihilations contain quarks and leptons together due to the leptoquark decays in our case. In other words, the region with $m_{S}>m_{L Q}$ can be constrained by indirect detection experiments too. The more general cases that the boost effects of leptoquarks cannot be ignored will be discussed in a future work. 


\subsection{Higgs data}

The decay rate of the Higgs boson into a pair of dark matter particles is

$$
\Gamma(h \rightarrow S S)=\frac{\lambda_{4}^{2} v^{2}}{32 \pi m_{h}} \sqrt{1-\frac{4 m_{S}^{2}}{m_{h}^{2}}} .
$$

The decay rate of the Higgs boson into a diphoton or a digluon is also modified due to leptoquarks, as given in eqs. (B.7) and (B.8). Corrections to $h \rightarrow W W, Z Z$ are small because they are already present at tree level in the SM, so we can ignore them. Then, the total Higgs decay width is modified to

$$
\Gamma_{h} \approx \Gamma_{h, \mathrm{SM}}+\Gamma(h \rightarrow S S)
$$

where $\Gamma_{h, \mathrm{SM}}=4 \mathrm{MeV}$ in the SM. The bound from invisible Higgs decay, $\mathrm{BR}(h \rightarrow S S)<$ 0.24 , leads to the following condition [90, 91],

$$
\mathrm{BR}(h \rightarrow S S)=\frac{\Gamma(h \rightarrow S S)}{\Gamma_{h}}<0.24 .
$$

The diphoton signal strength for gluon-fusion production is given by

$$
\mu_{\gamma \gamma}=R_{g g} R_{\gamma \gamma}
$$

where

$$
R_{g g}=\frac{\sigma(g g \rightarrow h)}{\sigma(g g \rightarrow h)_{\mathrm{SM}}}=\frac{\Gamma(h \rightarrow g g)}{\Gamma_{h} \cdot \mathrm{BR}(h \rightarrow g g)_{\mathrm{SM}}}, \quad R_{\gamma \gamma}=\frac{\Gamma(h \rightarrow \gamma \gamma)}{\Gamma_{h} \cdot \mathrm{BR}(h \rightarrow \gamma \gamma)_{\mathrm{SM}}} .
$$

The other visible decays, $h \rightarrow i j$, are similarly modified to $\mu_{i j}=R_{g g} R_{i j}$, through the modified total decay width of Higgs boson, with $R_{i j}=\mathrm{BR}(h \rightarrow i j) / \mathrm{BR}(h \rightarrow i j)_{\mathrm{SM}}=$ $\Gamma_{h, \mathrm{SM}} / \Gamma_{h}$. The measurements of $g g \rightarrow h \rightarrow \gamma \gamma$ show $\mu_{\gamma \gamma}=1.10_{-0.22}^{+0.23}$ from the combined fit of LHC $7 \mathrm{TeV}+8 \mathrm{TeV}$ data [92], and $\mu_{\gamma \gamma}=0.81_{-0.18}^{+0.19}$ and $\mu_{\gamma \gamma}=1.10_{-0.18}^{+0.20}$ from the ATLAS and CMS $13 \mathrm{TeV}$ data, respectively [93, 94].

In our model, as far as $\left|\lambda_{5}\right| \lesssim 10$, the decay rate into a diphoton or a digluon can be ignored, but the diphoton signal strength is modified by the enhanced total decay width of Higgs boson due to the invisible decay mode. This result can be read from figure 7 in the purple dot-dashed lines the region above which is excluded by Higgs invisible decay and in the green region which is excluded by the Higgs diphoton signal strength.

\section{Conclusions}

We have presented leptoquark models where scalar leptoquarks not only lead to the effective operators necessary for the $B$-meson anomalies but also become a portal to scalar dark matter through quartic couplings. We showed that the annihilations of dark matter into a leptoquark pair allow for a wide parameter space that is consistent with both the correct relic density and the XENON1T bound. These new annihilation channels lead to fourbody final states in cascade with quarks and leptons mixed, due to the leptoquark decays. Therefore, there is an interesting interplay between the cascade annihilations of dark matter and the leptoquark search channels at the LHC, which can be tested in the current and future experiments. 


\section{Acknowledgments}

The work is supported in part by Basic Science Research Program through the National Research Foundation of Korea (NRF) funded by the Ministry of Education, Science and Technology (NRF-2016R1A2B4008759). The work of TGR is supported in part by the Chung-Ang University Research Scholarship Grants in 2018.

\section{A Effective Hamiltonians for $B$-meson decays}

From eq. (3.2), we obtain the relevant Yukawa couplings for the singlet scalar leptoquark $S_{1}$ in components,

$$
\begin{aligned}
\mathcal{L}_{S_{1}}= & -\lambda_{3 j}\left(\overline{\left(t^{C}\right)_{R}} S_{1} l_{j L}-\overline{\left(b^{C}\right)_{R}} S_{1} \nu_{j L}\right)+\text { h.c. } \\
& -\lambda_{2 j}\left(\overline{\left(c^{C}\right)_{R}} S_{1} l_{j L}-\overline{\left(s^{C}\right)_{R}} S_{1} \nu_{j L}\right)+\text { h.c. }+\cdots
\end{aligned}
$$

Then, after integrating out the leptoquark $S_{1}$, we obtain the effective Hamiltonian relevant for $b \rightarrow c \tau \bar{\nu}_{\tau}$ as

$$
\begin{aligned}
\mathcal{H}_{b \rightarrow c \tau \bar{\nu}_{\tau}}^{S_{1}} & =-\frac{\lambda_{33}^{*} \lambda_{23}}{m_{S_{1}}^{2}}\left(\overline{\left(c^{C}\right)_{R}} \tau_{L}\right)\left(\bar{\nu}_{\tau L}\left(b^{C}\right)_{R}\right)+\text { h.c. } \\
& =-\frac{\lambda_{33}^{*} \lambda_{23}}{2 m_{S_{1}}^{2}}\left(\overline{\left(c^{C}\right)_{R}} \gamma^{\mu}\left(b^{C}\right)_{R}\right)\left(\bar{\nu}_{\tau L} \gamma_{\mu} \tau_{L}\right)+\text { h.c. } \\
& =-\frac{\lambda_{33}^{*} \lambda_{23}}{2 m_{S_{1}}^{2}}\left(\bar{b}_{L} \gamma^{\mu} c_{L}\right)\left(\bar{\nu}_{\tau L} \gamma_{\mu} \tau_{L}\right)+\text { h.c.. }
\end{aligned}
$$

where use is made of Fierz identity in the second line.

In particular, in MSSM, down-type squarks $\left(\tilde{b}_{R k}^{*}\right)$ [95] belong to singlet scalar leptoquarks. We introduce the R-parity violating (RPV) superpotential as follows,

$$
W \supset \lambda_{i j k}^{\prime} L_{i} Q_{j} D_{k}^{c}
$$

resulting in the component field Lagrangian for doublet scalar leptoquarks $S_{2} \equiv \tilde{u}_{L k}$ with $Y=+\frac{1}{6}$ or singlet scalar leptoquarks $S_{1}=\tilde{b}_{R k}^{*}$ with $Y=+\frac{1}{3}$ as

$$
\mathcal{L}_{R P V}=-\lambda_{i j k}^{\prime} L_{i} \tilde{Q}_{j} d_{k}^{c}+\text { h.c. }+\cdots
$$

Picking up the necessary terms for $R_{K^{(*)}}$ and $R_{D^{(*)}}$ anomalies, we get, in terms of two component spinors,

$$
\begin{aligned}
\mathcal{L}_{R P V}= & -\lambda_{j k 3}^{\prime} l_{j L} \tilde{u}_{L k} b^{c}-\lambda_{j k 2}^{\prime} l_{j L} \tilde{u}_{L k} s^{c} \\
& -\lambda_{j 3 k}^{\prime} \nu_{j L} b_{L} \tilde{b}_{R k}^{*}-\lambda_{j 2 k}^{\prime} l_{j L} c_{L} \tilde{b}_{R k}^{*}+\text { h.c. }+\cdots .
\end{aligned}
$$

Then, after integrating out the up-type squarks, $\tilde{u}_{L k}$, and down-type squarks, $\tilde{b}_{R k}^{*}$, we obtain the effective Hamiltonian for the semi-leptonic B-decays in terms of four-component 
spinors, as follows,

$$
\begin{aligned}
\mathcal{H}_{\mathrm{eff}}^{R P V} & =-\frac{\lambda_{2 k 3}^{\prime} \lambda_{2 k 2}^{\prime *}}{m_{\tilde{u}_{L k}}^{2}}\left(\bar{b}_{R} \mu_{L}\right)\left(\bar{\mu}_{L} s_{R}\right)-\frac{\lambda_{32 k}^{\prime} \lambda_{33 k}^{\prime *}}{m_{\tilde{d}_{R k}}^{2}}\left(\overline{\left(c^{C}\right)_{R}} \tau_{L}\right)\left(\bar{\nu}_{\tau L}\left(b^{C}\right)_{R}\right)+\text { h.c. } \\
& =-\frac{\lambda_{2 k 3}^{\prime} \lambda_{2 k 2}^{\prime *}}{2 m_{\tilde{u}_{L k}}^{2}}\left(\bar{b}_{R} \gamma^{\mu} s_{R}\right)\left(\bar{\mu}_{L} \gamma_{\mu} \mu_{L}\right)-\frac{\lambda_{32 k}^{\prime} \lambda_{33 k}^{\prime *}}{2 m_{\tilde{d}_{R k}}^{2}}\left(\bar{b}_{L} \gamma^{\mu} c_{L}\right)\left(\bar{\nu}_{\tau L} \gamma_{\mu} \tau_{L}\right)+\text { h.c.. }
\end{aligned}
$$

Therefore, the effective Hamiltonian for the $b$-to- $s$ transition is of the $(V+A)$ form, which was originally proposed to explain $R_{K}$ anomalies [96, 97] but is not consistent with $R_{K^{*}}$ anomalies as it favors $(V-A)$ form. On the other hand, the effective Hamiltonian for the $b$-to- $c$ transition is consistent with the $R_{D^{(*)}}$ anomalies [48, 98, 99].

From eq. (3.3), we obtain the relevant Yukawa couplings for the triplet leptoquark $S_{3}$ in components,

$$
\begin{aligned}
\mathcal{L}_{S_{3}}= & -\kappa_{3 j}\left(\sqrt{2} \overline{\left(t^{C}\right)_{R}} \phi_{3} \nu_{j L}-\overline{\left(t^{C}\right)_{R}} \phi_{2} l_{j L}-\overline{\left(b^{C}\right)_{R}} \phi_{2} \nu_{j L}-\sqrt{2} \overline{\left(b^{C}\right)_{R}} \phi_{1} l_{j L}\right)+\text { h.c. } \\
& -\kappa_{2 j}\left(\sqrt{2} \overline{\left(c^{C}\right)_{R}} \phi_{3} \nu_{j L}-\overline{\left(c^{C}\right)_{R}} \phi_{2} l_{j L}-\overline{\left(s^{C}\right)_{R}} \phi_{2} \nu_{j L}-\sqrt{2} \overline{\left(s^{C}\right)_{R}} \phi_{1} l_{j L}\right)+\text { h.c. }+\cdots
\end{aligned}
$$

Then, after integrating out the leptoquark $\phi_{1}$ with $Q=+\frac{4}{3}$, we obtain the effective Hamiltonian relevant for $b \rightarrow s \mu^{+} \mu^{-}$as

$$
\begin{aligned}
\mathcal{H}_{b \rightarrow s \mu^{+} \mu^{-}}^{S_{3}} & =-\frac{2 \kappa_{32}^{*} \kappa_{22}}{m_{\phi_{1}}^{2}}\left(\overline{\left(s^{C}\right)_{R}} \mu_{L}\right)\left(\bar{\mu}_{L}\left(b^{C}\right)_{R}\right)+\text { h.c. } \\
& =-\frac{\kappa_{32}^{*} \kappa_{22}}{m_{\phi_{1}}^{2}}\left(\overline{\left(s^{C}\right)_{R}} \gamma^{\mu}\left(b^{C}\right)_{R}\right)\left(\bar{\mu}_{L} \gamma_{\mu} \mu_{L}\right)+\text { h.c. } \\
& =-\frac{\kappa_{32}^{*} \kappa_{22}}{m_{\phi_{1}}^{2}}\left(\bar{b}_{L} \gamma^{\mu} s_{L}\right)\left(\bar{\mu}_{L} \gamma_{\mu} \mu_{L}\right)+\text { h.c. }
\end{aligned}
$$

Here, we note that use is made of the Fierz identity in the second line and $\overline{\left(s^{C}\right)_{R}} \gamma^{\mu}\left(b^{C}\right)_{R}=$ $b_{L}^{\dagger} \bar{\sigma}^{\mu} s_{L}=\bar{b}_{L} \gamma^{\mu} s_{L}$ is used in the third line.

The Yukawa couplings for the singlet scalar leptoquark also lead to effective Hamiltonian for $b \rightarrow s \nu_{i} \bar{\nu}_{j}$ as follows,

$$
\begin{aligned}
\mathcal{H}_{b \rightarrow s \nu_{i} \bar{\nu}_{j}}^{S_{1}} & =\frac{\lambda_{3 i}^{*} \lambda_{2 j}}{m_{S_{1}}^{2}}\left(\overline{\left(s^{c}\right)_{R}} \nu_{j L}\right)\left(\bar{\nu}_{i L}\left(b^{c}\right)_{R}\right)+\text { h.c. } \\
& =\frac{\lambda_{3 i}^{*} \lambda_{2 j}}{2 m_{S_{1}}^{2}}\left(\overline{\left(s^{c}\right)_{R}} \gamma^{\mu}\left(b^{c}\right)_{R}\right)\left(\bar{\nu}_{i L} \gamma_{\mu} \nu_{j L}\right)+\text { h.c. } \\
& =\frac{\lambda_{3 i}^{*} \lambda_{2 j}}{2 m_{S_{1}}^{2}}\left(\bar{b}_{L} \gamma^{\mu} s_{L}\right)\left(\bar{\nu}_{i L} \gamma_{\mu} \nu_{j L}\right)+\text { h.c. }
\end{aligned}
$$

A similar effective interactions can be obtained for the triplet scalar leptoquark, as discussed in the text. 


\section{B Effective interactions for dark matter and Higgs boson due to lepto- quark loops}

For heavy leptoquarks, we the effective interactions between scalar dark matter and SM gauge bosons, induced by leptoquarks, as follows,

$$
\mathcal{L}_{S, \mathrm{eff}}=D_{3} S^{2} G_{\mu \nu} G^{\mu \nu}+D_{2} S^{2} W_{\mu \nu} W^{\mu \nu}+D_{1} S^{2} F_{Y \mu \nu} F^{Y \mu \nu}
$$

where

$$
\begin{aligned}
D_{3} & =\frac{\alpha_{S} \lambda_{3}}{32 \pi m_{L Q}^{2}} N_{L Q} l_{3}\left(S_{L Q}\right) A_{0}(y), \\
D_{2} & =\frac{\alpha \lambda_{3}}{32 \pi m_{L Q}^{2}} N_{c} l_{2}\left(S_{L Q}\right) A_{0}(y), \\
D_{1} & =\frac{\alpha_{Y} \lambda_{3}}{32 \pi m_{L Q}^{2}} N_{c} N_{L Q} Y_{L Q}^{2} A_{0}(y)
\end{aligned}
$$

with

$$
\begin{aligned}
A_{0}(y) & =-y^{-2}[y-f(y)], \\
f(y) & = \begin{cases}\arcsin ^{2} \sqrt{y}, & y \leq 1 \\
-\frac{1}{4}\left[\ln \frac{1+\sqrt{1-y^{-1}}}{1-\sqrt{1-y^{-1}}}-i \pi\right], & y>1\end{cases}
\end{aligned}
$$

and $y \equiv m_{S}^{2} / m_{L Q}^{2}$. Here, $l_{2,3}\left(S_{L Q}\right)$ are the Dynkin indices of $S_{L Q}$ under $\mathrm{SU}(2)_{L}$ and $\mathrm{SU}(3)_{c}$, respectively, i.e. $l_{3}\left(S_{1,3}\right)=\frac{1}{2}, l_{2}\left(S_{1}\right)=0$, and $l_{2}\left(S_{3}\right)=2$, and $N_{L Q}=1,3$ for $S_{L Q}=S_{1}, S_{3}$, respectively.

Moreover, leptoquark couplings to the SM Higgs can modify the decay rates of Higgs boson into a diphoton or a digluon, as follows,

$$
\begin{aligned}
\Gamma(h \rightarrow \gamma \gamma)= & \frac{G_{F} \alpha_{\mathrm{em}}^{2} m_{h}^{3}}{128 \sqrt{2} \pi^{3}} \mid \sum_{f} N_{c} Q_{f}^{2} A_{1 / 2}\left(x_{f}\right)+A_{1}\left(x_{W}\right) \\
& +\left.N_{c} g_{L Q} \sum_{i=1, \cdots, N_{L Q}} Q_{L Q}^{2} A_{0}\left(x_{L Q}\right)\right|^{2}, \\
\Gamma(h \rightarrow g g)= & \frac{G_{F} \alpha_{s}^{2} m_{h}^{3}}{36 \sqrt{2} \pi^{3}}\left|\frac{3}{4} \sum_{f} A_{1 / 2}\left(x_{f}\right)+\frac{3}{4} N_{L Q} g_{L Q} A_{0}\left(x_{L Q}\right)\right|^{2}
\end{aligned}
$$

where $g_{L Q} \equiv \lambda_{5} v^{2} /\left(2 m_{L Q}^{2}\right), x_{i}=m_{h}^{2} /\left(4 m_{i}^{2}\right)$ and the loop functions are

$$
\begin{aligned}
A_{1 / 2}(x) & =2 x^{-2}[x+(x-1) f(x)], \\
A_{1}(x) & =-x^{-2}\left[2 x^{2}+3 x+3(2 x-1) f(x)\right] .
\end{aligned}
$$


Open Access. This article is distributed under the terms of the Creative Commons Attribution License (CC-BY 4.0), which permits any use, distribution and reproduction in any medium, provided the original author(s) and source are credited.

\section{References}

[1] LHCb collaboration, Test of lepton universality using $B^{+} \rightarrow K^{+} \ell^{+} \ell^{-}$decays, Phys. Rev. Lett. 113 (2014) 151601 [arXiv: 1406.6482] [INSPIRE].

[2] S. Bifani, seminar at CERN (2017), https://indico.cern.ch/event/580620/.

[3] LHCb collaboration, S. Bifani, Status of New Physics searches with $b \rightarrow s \ell^{+} \ell^{-}$transitions $\mathrm{LHCb}$, in proceedings of 52nd Rencontres de Moriond on Electroweak Interactions and Unified Theories, La Thuile, Italy, 18-25 March 2017, pp. 197-202, arXiv:1705.02693 [INSPIRE].

[4] LHCb collaboration, Test of lepton universality with $B^{0} \rightarrow K^{* 0} \ell^{+} \ell^{-}$decays, JHEP 08 (2017) 055 [arXiv: 1705. 05802] [INSPIRE].

[5] LHCb collaboration, Measurement of Form-Factor-Independent Observables in the Decay $B^{0} \rightarrow K^{* 0} \mu^{+} \mu^{-}$, Phys. Rev. Lett. 111 (2013) 191801 [arXiv:1308.1707] [INSPIRE].

[6] LHCb collaboration, Angular analysis of the $B^{0} \rightarrow K^{* 0} \mu^{+} \mu^{-}$decay using $3 \mathrm{fb}^{-1}$ of integrated luminosity, JHEP 02 (2016) 104 [arXiv:1512.04442] [INSPIRE].

[7] BABAR collaboration, J.P. Lees et al., Evidence for an excess of $\bar{B} \rightarrow D^{(*)} \tau^{-} \bar{\nu}_{\tau}$ decays, Phys. Rev. Lett. 109 (2012) 101802 [arXiv:1205.5442] [INSPIRE].

[8] BaBAr collaboration, J.P. Lees et al., Measurement of an Excess of $\bar{B} \rightarrow D^{(*)} \tau^{-} \bar{\nu}_{\tau}$ Decays and Implications for Charged Higgs Bosons, Phys. Rev. D 88 (2013) 072012 [arXiv:1303.0571] [INSPIRE].

[9] Belle collaboration, M. Huschle et al., Measurement of the branching ratio of $\bar{B} \rightarrow D^{(*)} \tau^{-} \bar{\nu}_{\tau}$ relative to $\bar{B} \rightarrow D^{(*)} \ell^{-} \bar{\nu}_{\ell}$ decays with hadronic tagging at Belle, Phys. Rev. $\mathbf{D}$ 92 (2015) 072014 [arXiv: 1507.03233] [INSPIRE].

[10] Belle collaboration, A. Abdesselam et al., Measurement of the branching ratio of $\bar{B}^{0} \rightarrow D^{*+} \tau^{-} \bar{\nu}_{\tau}$ relative to $\bar{B}^{0} \rightarrow D^{*+} \ell^{-} \bar{\nu}_{\ell}$ decays with a semileptonic tagging method, in proceedings of 51st Rencontres de Moriond on Electroweak Interactions and Unified Theories, La Thuile, Italy, 12-19 March 2016, arXiv:1603.06711 [INSPIRE].

[11] LHCb collaboration, Measurement of the ratio of branching fractions $\mathcal{B}\left(\bar{B}^{0} \rightarrow D^{*+} \tau^{-} \bar{\nu}_{\tau}\right) / \mathcal{B}\left(\bar{B}^{0} \rightarrow D^{*+} \mu^{-} \bar{\nu}_{\mu}\right)$, Phys. Rev. Lett. 115 (2015) 111803 [Erratum ibid. 115 (2015) 159901] [arXiv: 1506.08614] [INSPIRE].

[12] A. Bharucha, D.M. Straub and R. Zwicky, $B \rightarrow V \ell^{+} \ell^{-}$in the Standard Model from light-cone sum rules, JHEP 08 (2016) 098 [arXiv: 1503.05534] [INSPIRE].

[13] M. Ciuchini, M. Fedele, E. Franco, S. Mishima, A. Paul, L. Silvestrini et al., $B \rightarrow K^{*} \ell^{+} \ell^{-}$ decays at large recoil in the Standard Model: a theoretical reappraisal, JHEP 06 (2016) 116 [arXiv: 1512.07157] [INSPIRE].

[14] S. Neshatpour, V.G. Chobanova, T. Hurth, F. Mahmoudi and D. Martinez Santos, Direct comparison of global fits to the $B \rightarrow K^{*} \mu^{+} \mu^{-}$data assuming hadronic corrections or new physics, in proceedings of 52nd Rencontres de Moriond on QCD and High Energy Interactions, La Thuile, Italy, 25 March - 1 April 2017, pp. 87-90, arXiv:1705.10730 [INSPIRE]. 
[15] A. Arbey, T. Hurth, F. Mahmoudi and S. Neshatpour, Hadronic and New Physics Contributions to $B \rightarrow K^{*} \ell^{+} \ell^{-}$, arXiv:1806.02791 [INSPIRE].

[16] J. Albrecht, F. Bernlochner, M. Kenzie, S. Reichert, D. Straub and A. Tully, Future prospects for exploring present day anomalies in flavour physics measurements with Belle II and $L H C b$, arXiv: 1709.10308 [INSPIRE].

[17] XENON collaboration, E. Aprile et al., Dark Matter Search Results from a One Tonne $\times$ Year Exposure of XENON1T, Phys. Rev. Lett. 121 (2018) 111302 [arXiv:1805.12562] [INSPIRE].

[18] XENON collaboration, E. Aprile et al., First Dark Matter Search Results from the XENON1T Experiment, Phys. Rev. Lett. 119 (2017) 181301 [arXiv:1705. 06655] [InSPIRE].

[19] PandaX-II collaboration, X. Cui et al., Dark Matter Results From 54-Ton-Day Exposure of PandaX-II Experiment, Phys. Rev. Lett. 119 (2017) 181302 [arXiv:1708.06917] [INSPIRE].

[20] SuperCDMS collaboration, R. Agnese et al., Results from the Super Cryogenic Dark Matter Search Experiment at Soudan, Phys. Rev. Lett. 120 (2018) 061802 [arXiv:1708.08869] [INSPIRE].

[21] LUX collaboration, D.S. Akerib et al., Results from a search for dark matter in the complete LUX exposure, Phys. Rev. Lett. 118 (2017) 021303 [arXiv: 1608. 07648] [INSPIRE].

[22] DARWIN collaboration, J. Aalbers et al., DARWIN: towards the ultimate dark matter detector, JCAP 11 (2016) 017 [arXiv: 1606.07001] [INSPIRE].

[23] LZ collaboration, D.S. Akerib et al., LUX-ZEPLIN (LZ) Conceptual Design Report, arXiv: 1509.02910 [INSPIRE].

[24] W. Buchmüller, R. Ruckl and D. Wyler, Leptoquarks in lepton-quark collisions, Phys. Lett. B 191 (1987) 442 [Erratum ibid. B 448 (1999) 320] [INSPIRE].

[25] I. Doršner, S. Fajfer, A. Greljo, J.F. Kamenik and N. Košnik, Physics of leptoquarks in precision experiments and at particle colliders, Phys. Rept. 641 (2016) 1 [arXiv:1603.04993] [INSPIRE].

[26] A. Crivellin, D. Müller and T. Ota, Simultaneous explanation of $R_{D^{(*)}}$ and $b \rightarrow s \mu^{+} \mu^{-}$: the last scalar leptoquarks standing, JHEP 09 (2017) 040 [arXiv:1703.09226] [INSPIRE].

[27] G. Hiller and I. Nisandzic, $R_{K}$ and $R_{K^{*}}$ beyond the standard model, Phys. Rev. D 96 (2017) 035003 [arXiv: 1704.05444] [INSPIRE].

[28] D. Buttazzo, A. Greljo, G. Isidori and D. Marzocca, B-physics anomalies: a guide to combined explanations, JHEP 11 (2017) 044 [arXiv: 1706.07808] [INSPIRE].

[29] M. Bauer and M. Neubert, Minimal Leptoquark Explanation for the $R_{D^{(*)}}, R_{K}$ and $(g-2)_{g}$ Anomalies, Phys. Rev. Lett. 116 (2016) 141802 [arXiv:1511.01900] [INSPIRE].

[30] C.-H. Chen, T. Nomura and H. Okada, Excesses of muon $g-2, R_{D^{(*)}}$ and $R_{K}$ in a leptoquark model, Phys. Lett. B 774 (2017) 456 [arXiv:1703.03251] [INSPIRE].

[31] S. Matsuzaki, K. Nishiwaki and R. Watanabe, Phenomenology of flavorful composite vector bosons in light of $B$ anomalies, JHEP 08 (2017) 145 [arXiv:1706.01463] [INSPIRE].

[32] J. Kumar, D. London and R. Watanabe, Combined Explanations of the $b \rightarrow s \mu^{+} \mu^{-}$and $b \rightarrow c \tau^{-} \bar{\nu}$ Anomalies: a General Model Analysis, arXiv: 1806.07403 [INSPIRE].

[33] N. Assad, B. Fornal and B. Grinstein, Baryon Number and Lepton Universality Violation in Leptoquark and Diquark Models, Phys. Lett. B 777 (2018) 324 [arXiv:1708.06350] [INSPIRE]. 
[34] L. Di Luzio, A. Greljo and M. Nardecchia, Gauge leptoquark as the origin of B-physics anomalies, Phys. Rev. D 96 (2017) 115011 [arXiv:1708.08450] [INSPIRE].

[35] L. Calibbi, A. Crivellin and T. Li, A model of vector leptoquarks in view of the B-physics anomalies, arXiv:1709.00692 [INSPIRE].

[36] M. Bordone, C. Cornella, J. Fuentes-Martin and G. Isidori, A three-site gauge model for flavor hierarchies and flavor anomalies, Phys. Lett. B 779 (2018) 317 [arXiv:1712.01368] [INSPIRE].

[37] M. Blanke and A. Crivellin, B Meson Anomalies in a Pati-Salam Model within the Randall-Sundrum Background, Phys. Rev. Lett. 121 (2018) 011801 [arXiv:1801.07256] [INSPIRE].

[38] D. Bečirević, I. Doršner, S. Fajfer, N. Košnik, D.A. Faroughy and O. Sumensari, Scalar leptoquarks from grand unified theories to accommodate the B-physics anomalies, Phys. Rev. D 98 (2018) 055003 [arXiv: 1806.05689] [INSPIRE].

[39] B. Capdevila, A. Crivellin, S. Descotes-Genon, J. Matias and J. Virto, Patterns of New Physics in $b \rightarrow s \ell^{+} \ell^{-}$transitions in the light of recent data, JHEP 01 (2018) 093 [arXiv: 1704.05340] [INSPIRE].

[40] HFLAV collaboration, Y. Amhis et al., Averages of b-hadron, c-hadron and $\tau$-lepton properties as of summer 2016, Eur. Phys. J. C 77 (2017) 895 [arXiv:1612.07233] [InSPIRE].

[41] HPQCD collaboration, H. Na, C.M. Bouchard, G.P. Lepage, C. Monahan and J. Shigemitsu, $B \rightarrow D l \nu$ form factors at nonzero recoil and extraction of $\left|V_{c b}\right|$, Phys. Rev. D 92 (2015) 054510 [Erratum ibid. D 93 (2016) 119906] [arXiv: 1505.03925] [INSPIRE].

[42] S. Fajfer, J.F. Kamenik and I. Nisandzic, On the $B \rightarrow D^{*} \tau \bar{\nu}_{\tau}$ Sensitivity to New Physics, Phys. Rev. D 85 (2012) 094025 [arXiv: 1203.2654] [inSPIRE].

[43] F.U. Bernlochner, Z. Ligeti, M. Papucci and D.J. Robinson, Combined analysis of semileptonic $B$ decays to $D$ and $D^{*}: R\left(D^{(*)}\right),\left|V_{c b}\right|$ and new physics, Phys. Rev. D 95 (2017) 115008 [Erratum ibid. D 97 (2018) 059902] [arXiv:1703.05330] [INSPIRE].

[44] D. Bigi and P. Gambino, Revisiting B $\rightarrow$ D $\ell$, Phys. Rev. D 94 (2016) 094008 [arXiv: 1606.08030] [INSPIRE].

[45] MILC collaboration, J.A. Bailey et al., $B \rightarrow D \ell \nu$ form factors at nonzero recoil and $-V_{c b}-$ from 2+1-flavor lattice QCD, Phys. Rev. D 92 (2015) 034506 [arXiv:1503.07237] [InSPIRE].

[46] D. Bigi, P. Gambino and S. Schacht, $R\left(D^{*}\right),\left|V_{c b}\right|$ and the Heavy Quark Symmetry relations between form factors, JHEP 11 (2017) 061 [arXiv:1707.09509] [INSPIRE].

[47] S. Jaiswal, S. Nandi and S.K. Patra, Extraction of $\left|V_{c b}\right|$ from $B \rightarrow D^{(*)} \ell \nu_{\ell}$ and the Standard Model predictions of $R\left(D^{(*)}\right)$, JHEP 12 (2017) 060 [arXiv:1707.09977] [INSPIRE].

[48] W. Altmannshofer, P. Bhupal Dev and A. Soni, $R_{D^{(*)}}$ anomaly: A possible hint for natural supersymmetry with R-parity violation, Phys. Rev. D 96 (2017) 095010 [arXiv:1704.06659] [INSPIRE].

[49] L. Di Luzio and M. Nardecchia, What is the scale of new physics behind the B-flavour anomalies?, Eur. Phys. J. C 77 (2017) 536 [arXiv:1706.01868] [INSPIRE].

[50] CMS collaboration, Search for the pair production of third-generation squarks with two-body decays to a bottom or charm quark and a neutralino in proton-proton collisions at $\sqrt{s}=13$ TeV, Phys. Lett. B 778 (2018) 263 [arXiv:1707.07274] [InSPIRE]. 
[51] ATLAS collaboration, Search for Supersymmetry in events with b-tagged jets and missing transverse energy in pp collisions at $\sqrt{s}=13 \mathrm{TeV}$ with the ATLAS detector,

ATLAS-CONF-2017-038 (2017).

[52] CMS collaboration, Search for supersymmetry in multijet events with missing transverse momentum in proton-proton collisions at 13 TeV, Phys. Rev. D 96 (2017) 032003 [arXiv: 1704.07781] [INSPIRE].

[53] ATLAS collaboration, Search for squarks and gluinos in final states with jets and missing transverse momentum using $36 \mathrm{fb}^{-1}$ of $\sqrt{\mathrm{s}}=13 \mathrm{TeV}$ pp collision data with the ATLAS detector, ATLAS-CONF-2017-022 (2017).

[54] ATLAS collaboration, A search for B-L R-parity-violating scalar tops in $\sqrt{s}=13 \mathrm{TeV} p p$ collisions with the ATLAS experiment, ATLAS-CONF-2017-036 (2017).

[55] CMS collaboration, Search for pair production of first and second generation leptoquarks in proton-proton collisions at $\sqrt{s}=8 \mathrm{TeV}$, Phys. Rev. D 93 (2016) 032004 [arXiv:1509.03744] [INSPIRE].

[56] CMS Collaboration, Search for leptoquarks coupling to third generation quarks, CMS-PAS-B2G-16-027 (2017).

[57] ATLAS collaboration, Searches for scalar leptoquarks in pp collisions at $\sqrt{s}=8 \mathrm{TeV}$ with the ATLAS detector, Eur. Phys. J. C 76 (2016) 5 [arXiv:1508.04735] [inSPIRE].

[58] CMS collaboration, Search for top squark pair production in pp collisions at $\sqrt{s}=13 \mathrm{TeV}$ using single lepton events, JHEP 10 (2017) 019 [arXiv:1706.04402] [INSPIRE].

[59] ATLAS collaboration, Search for top-squark pair production in final states with one lepton, jets and missing transverse momentum using $36 \mathrm{fb}^{-1}$ of $\sqrt{s}=13 \mathrm{TeV}$ pp collision data with the ATLAS detector, JHEP 06 (2018) 108 [arXiv:1711.11520] [INSPIRE].

[60] L. Bian, H.M. Lee and C.B. Park, B-meson anomalies and Higgs physics in flavored $U(1)^{\prime}$ model, Eur. Phys. J. C 78 (2018) 306 [arXiv:1711.08930] [inSPIRE].

[61] L. Bian, S.-M. Choi, Y.-J. Kang and H.M. Lee, A minimal flavored U(1)' for B-meson anomalies, Phys. Rev. D 96 (2017) 075038 [arXiv:1707.04811] [INSPIRE].

[62] A.K. Alok, B. Bhattacharya, D. Kumar, J. Kumar, D. London and S.U. Sankar, New physics in $b \rightarrow s \mu^{+} \mu^{-}$: Distinguishing models through CP-violating effects, Phys. Rev. D 96 (2017) 015034 [arXiv: 1703.09247] [INSPIRE].

[63] Belle collaboration, J. Grygier et al., Search for $B \rightarrow h \nu \bar{\nu}$ decays with semileptonic tagging at Belle, Phys. Rev. D 96 (2017) 091101 [Addendum ibid. D 97 (2018) 099902] [arXiv: 1702.03224] [INSPIRE].

[64] A.J. Buras, J. Girrbach-Noe, C. Niehoff and D.M. Straub, $B \rightarrow K^{(*)} \nu \bar{\nu}$ decays in the Standard Model and beyond, JHEP 02 (2015) 184 [arXiv: 1409.4557] [INSPIRE].

[65] D.A. Faroughy, A. Greljo and J.F. Kamenik, Confronting lepton flavor universality violation in B decays with high-p $p_{T}$ tau lepton searches at LHC, Phys. Lett. B 764 (2017) 126 [arXiv: 1609.07138] [INSPIRE].

[66] B. Diaz, M. Schmaltz and Y.-M. Zhong, The leptoquark Hunter's guide: Pair production, JHEP 10 (2017) 097 [arXiv: 1706. 05033] [INSPIRE].

[67] G. Hiller, D. Loose and I. Nišandžić, Flavorful leptoquarks at hadron colliders, Phys. Rev. D 97 (2018) 075004 [arXiv: 1801.09399] [INSPIRE]. 
[68] I. Doršner and A. Greljo, Leptoquark toolbox for precision collider studies, JHEP 05 (2018) 126 [arXiv: 1801.07641 ] [INSPIRE].

[69] Muon G-2 collaboration, G.W. Bennett et al., Final Report of the Muon E821 Anomalous Magnetic Moment Measurement at BNL, Phys. Rev. D 73 (2006) 072003 [hep-ex/0602035] [INSPIRE].

[70] Particle Data Group collaboration, C. Patrignani et al., Review of Particle Physics, Chin. Phys. C 40 (2016) 100001 [inSPIRE].

[71] BaBAR collaboration, B. Aubert et al., Searches for Lepton Flavor Violation in the Decays $\tau^{ \pm} \rightarrow e^{ \pm} \gamma$ and $\tau^{ \pm} \rightarrow \mu^{ \pm} \gamma$, Phys. Rev. Lett. 104 (2010) 021802 [arXiv:0908.2381] [INSPIRE].

[72] M.J. Baker et al., The Coannihilation Codex, JHEP 12 (2015) 120 [arXiv:1510.03434] [INSPIRE].

[73] M. Bauer and M. Neubert, Flavor anomalies, the $750 \mathrm{GeV}$ diphoton excess and a dark matter candidate, Phys. Rev. D 93 (2016) 115030 [arXiv:1512.06828] [InSPIRE].

[74] F.S. Queiroz, K. Sinha and A. Strumia, Leptoquarks, Dark Matter and Anomalous LHC Events, Phys. Rev. D 91 (2015) 035006 [arXiv:1409.6301] [INSPIRE].

[75] B. Allanach, A. Alves, F.S. Queiroz, K. Sinha and A. Strumia, Interpreting the CMS $\ell^{+} \ell^{-} j j \mathbb{F}_{T}$ Excess with a Leptoquark Model, Phys. Rev. D 92 (2015) 055023 [arXiv: 1501.03494] [INSPIRE].

[76] S.M. Choi, Y.J. Kang, H.M. Lee and T.G. Ro, WIMP dark matter via lepto-quark portal, to appear.

[77] J.M. Cline, $130 \mathrm{GeV}$ dark matter and the Fermi gamma-ray line, Phys. Rev. D 86 (2012) 015016 [arXiv: 1205. 2688] [INSPIRE].

[78] H.M. Lee, M. Park and W.-I. Park, Fermi Gamma Ray Line at 130 GeV from Axion-Mediated Dark Matter, Phys. Rev. D 86 (2012) 103502 [arXiv:1205.4675] [INSPIRE].

[79] H.M. Lee, M. Park and W.-I. Park, Axion-mediated dark matter and Higgs diphoton signal, JHEP 12 (2012) 037 [arXiv: 1209.1955] [INSPIRE].

[80] H.M. Lee, M. Park and V. Sanz, Interplay between Fermi gamma-ray lines and collider searches, JHEP 03 (2013) 052 [arXiv: 1212.5647] [INSPIRE].

[81] S.-M. Choi, Y.-J. Kang and H.M. Lee, Diphoton resonance confronts dark matter, JHEP 07 (2016) 030 [arXiv : 1605.04804] [INSPIRE].

[82] J. Hisano, K. Ishiwata, N. Nagata and M. Yamanaka, Direct Detection of Vector Dark Matter, Prog. Theor. Phys. 126 (2011) 435 [arXiv: 1012.5455] [INSPIRE].

[83] FERMI-LAT collaboration, M. Ackermann et al., Updated search for spectral lines from Galactic dark matter interactions with pass 8 data from the Fermi Large Area Telescope, Phys. Rev. D 91 (2015) 122002 [arXiv:1506.00013] [INSPIRE].

[84] HESS collaboration, H. Abdallah et al., Search for $\gamma$-Ray Line Signals from Dark Matter Annihilations in the Inner Galactic Halo from 10 Years of Observations with H.E.S.S., Phys. Rev. Lett. 120 (2018) 201101 [arXiv: 1805.05741] [INSPIRE].

[85] H.E.S.S. collaboration, H. Abdalla et al., H.E.S.S. Limits on Linelike Dark Matter Signatures in the $100 \mathrm{GeV}$ to $2 \mathrm{TeV}$ Energy Range Close to the Galactic Center, Phys. Rev. Lett. 117 (2016) 151302 [arXiv: 1609.08091] [INSPIRE]. 
[86] Fermi-LAT collaboration, M. Ackermann et al., Searching for Dark Matter Annihilation from Milky Way Dwarf Spheroidal Galaxies with Six Years of Fermi Large Area Telescope Data, Phys. Rev. Lett. 115 (2015) 231301 [arXiv:1503.02641] [INSPIRE].

[87] H.E.S.S. collaboration, H. Abdallah et al., Search for dark matter annihilations towards the inner Galactic halo from 10 years of observations with H.E.S.S, Phys. Rev. Lett. 117 (2016) 111301 [arXiv: 1607.08142] [INSPIRE].

[88] AMS collaboration, M. Aguilar et al., Antiproton Flux, Antiproton-to-Proton Flux Ratio and Properties of Elementary Particle Fluxes in Primary Cosmic Rays Measured with the Alpha Magnetic Spectrometer on the International Space Station, Phys. Rev. Lett. 117 (2016) 091103 [INSPIRE].

[89] A. Cuoco, J. Heisig, M. Korsmeier and M. Krämer, Constraining heavy dark matter with cosmic-ray antiprotons, JCAP 04 (2018) 004 [arXiv: 1711.05274] [INSPIRE].

[90] ATLAS collaboration, Constraints on new phenomena via Higgs boson couplings and invisible decays with the ATLAS detector, JHEP 11 (2015) 206 [arXiv:1509.00672] [INSPIRE].

[91] CMS collaboration, Searches for invisible decays of the Higgs boson in pp collisions at $\sqrt{s}=$ 7, 8 and 13 TeV, JHEP 02 (2017) 135 [arXiv:1610.09218] [InSPIRE].

[92] ATLAS, CMS collaboration, G. Aad et al., Measurements of the Higgs boson production and decay rates and constraints on its couplings from a combined ATLAS and CMS analysis of the LHC pp collision data at $\sqrt{s}=7$ and 8 TeV, JHEP 08 (2016) 045 [arXiv: 1606. 02266] [INSPIRE].

[93] ATLAS collaboration, Measurements of Higgs boson properties in the diphoton decay channel with $36 \mathrm{fb}^{-1}$ of pp collision data at $\sqrt{s}=13 \mathrm{TeV}$ with the ATLAS detector, Phys. Rev. D 98 (2018) 052005 [arXiv: 1802.04146] [InSPIRE].

[94] CMS collaboration, Measurements of Higgs boson properties in the diphoton decay channel in proton-proton collisions at $\sqrt{s}=13 \mathrm{TeV}$, arXiv: 1804.02716 [INSPIRE].

[95] E.J. Chun, S. Jung, H.M. Lee and S.C. Park, Stop and Sbottom LSP with R-parity Violation, Phys. Rev. D 90 (2014) 115023 [arXiv:1408.4508] [InSPIRE].

[96] G. Hiller and M. Schmaltz, $R_{K}$ and future $b \rightarrow$ sll physics beyond the standard model opportunities, Phys. Rev. D 90 (2014) 054014 [arXiv:1408.1627] [INSPIRE].

[97] S. Biswas, D. Chowdhury, S. Han and S.J. Lee, Explaining the lepton non-universality at the LHCb and CMS within a unified framework, JHEP 02 (2015) 142 [arXiv:1409.0882] [INSPIRE].

[98] N.G. Deshpande and X.-G. He, Consequences of R-parity violating interactions for anomalies in $\bar{B} \rightarrow D^{(*)} \tau \bar{\nu}$ and $b \rightarrow s \mu^{+} \mu^{-}$, Eur. Phys. J. C 77 (2017) 134 [arXiv:1608.04817] [INSPIRE].

[99] D. Das, C. Hati, G. Kumar and N. Mahajan, Scrutinizing R-parity violating interactions in light of $R_{K^{(*)}}$ data, Phys. Rev. D 96 (2017) 095033 [arXiv: 1705.09188] [INSPIRE]. 